(C) 2020 A. M. SUMAROKOV, A. M. ZAMOROKA

\title{
SPATIAL PATTERNS AND HABITAT DIFFERENTIATION OF THE BEETLE (INSECTA: COLEOPTERA) FAUNA OF PROTECTED AREAS IN THE EASTERN CARPATHIANS (IVANO-FRANKIVSK REGION, UKRAINE)
}

\begin{abstract}
Сумароков, О. М., Заморока, А. М. Просторовий розподіл та оселищна диференціація фауни твердокрилих (Insecta: Coleoptera) природно-заповідних територій Східних Карпат (Івано-Франківська область, Україна). Вісті Харківського ентомологічного товариства. 2020. T. XXVIII, вип. 2. С. 5-25. DOI: 10.36016/KhESG-2020-28-2-1.

Попри те, що твердокрилих на території Східних Карпат вивчають ось уже 200 років, до сьогодні залишаються достеменно невідомими ні загальна кількість видів, ані остаточний склад фауни терену. Однак, в умовах природоохоронної діяльності першочерговим завданням $€$ виявлення гарячих точок біорозмаїття і з’ясування причинно-наслідкових зв'язків, що зумовлюють їхнє існування. У цій канві ми здійснили дослідження фауни твердокрилих уздовж висотного градієнта на природно-заповідних територіях Східних Карпат. У результаті досліджень нами виявлено 595 видів жуків із 37 родин, яких проаналізовано за критеріями просторового розподілу та оселищної диференціації. Установлено, що $\beta$-розмаїття твердокрилих збіднюється зі зростанням відносних висот від 327 видів на висоті 325 м н. р. м. до 48 видів на висоті 1800 м н. р. м. При цьому, фауністична матриця регіону складена лісовими видами, на фоні яких виразно виокремлюються фауни лучних степів у долині Дністра та альпійських лук на хребті Чорногора. Фауна твердокрилих є високо диференційованою стосовно оселищ, виявляючи низьку подібність між видовими спектрами в них. Функціональні зв’язки розмаїття твердокрилих у екосистемах виявляються у різних типах трансляції енергії в межах трофічних ланцюгів. Зокрема, ми виявили продуцентно-консументний тип трансляції у лучних екосистемах і редуцентноконсументний - у лісових. Це вказує на різний рівень стабільності екосистем, а відтак і загроз для них. Вважаємо, що функціональне розмаїття твердокрилих $є$ індикатором того, що лісові екосистеми є більш стабільними із вищою екологічною ємністю, а лучні - більш уразливими до антропогенних і природних чинників. У світлі результатів наших досліджень існує потреба забезпечення особливого охоронного режиму для альпійських і лучно-степових оселищ твердокрилих регіону.
\end{abstract}

Ключові слова: висотний градієнт, $\beta$-розмаїття, функціональне розмаїття, екосистемна ємність.

4 рис., 2 табл., 52 назв.

Сумароков, А. М., Заморока, А. М. Пространственное распределение и стациальная дифференциация фауны жесткокрылых (Insecta: Coleoptera) охраняемых территорий Восточных Карпат (Ивано-Франковская область, Украина). Известия Харьковского энтомологического общества. 2020. T. XXVIII, вып. 2. С. 5-25. DOI: 10.36016/KhESG-2020-28-2-1.

Несмотря на то, что жесткокрылых Восточных Карпат изучают вот уже 200 лет, до настоящего времени остаются доподлинно неизвестными как общее количество видов, так и полный состав фауны данной территории. Однако, в условиях природоохранной деятельности первоочередным заданием является выявление горячих точек биоразнообразия и причин их возникновения. В этом русле нами проведены исследования фауны жесткокрылых вдоль высотного градиента на природоохранных территориях Восточных Карпат. В результате исследований нами обнаружено 595 видов жуков из 37 семейств, которых проанализировано по критериям пространственного распределения и стациальной дифференциации. Установлено, что $\beta$-разнообразие жесткокрылых обедняется с возрастанием относительных высот с 327 видов на высоте 325 м н. у. м. до 48 видов на высоте 1800 м н. у. м. При этом, фаунистическая матрица региона составлена лесными видами, на фоне которых отчётливо выделяются фауны луговых степей в долине Днестра и альпийских лугов на хребте Черногора. Фауна жесткокрылых высоко дифференцирована относительно стаций, проявляя низкое сходство между спектрами видов в них. Функциональные связи разнообразия жесткокрылых в экосистемах проявляются в разных типах трансляции энергии в пределах трофических цепей. В частности, мы обнаружили продуцентноконсументный тип трансляции в луговых экосистемах и редуцентно-консументный - в лесных экосистемах. Это указывает на разный уровень стабильности экосистем, а, следовательно, и угроз для них. Считаем, что функциональное разнообразие жесткокрылых выступает индикатором того, что лесные экосистемы являются более стабильными с высшей экологической ёмкостью, а луговые - более уязвимыми к антропогенным и природным факторам. В свете результатов наших исследований существует необходимость обеспечения особого охранного режима для альпийских и луго-степных местообитаний жесткокрылых региона.

4 рис., 2 табл., 52 назв.

Ключевые слова: высотный градиент, $\beta$-разнообразие, функциональное разнообразие, экосистемная ёмкость.

Sumarokov, A. M., Zamoroka, A. M. Spatial patterns and habitat differentiation of the beetle (Insecta: Coleoptera) fauna of protected areas in the Eastern Carpathians (Ivano-Frankivsk Region, Ukraine). The Kharkov Entomological Society Gazette. 2020. Vol. XXVIII, iss. 2. P. 5-25. DOI: 10.36016/KhESG-2020-28-2-1.

Despite the beetles in the Eastern Carpathians have been studied for 200 years, a complete and exhaustive list of beetles has not been done yet. Nevertheless, the main tasks of conservation activity are to identify biodiversity hot spots and to determine the causes of their existence. In this outline, we studied the Coleoptera fauna of protected areas in the Eastern Carpathians. We identified 595 species of beetles from 37 families, which analyzed by the criteria of spatial patterns and habitat differentiation. We found ascending altitudinal gradient in species richness from 327 species at $325 \mathrm{~m}$ a. s. 1 . to 48 species at $1,800 \mathrm{~m}$ a. s. l.. The sylvatic species of Coleoptera predominate in throughout the

Sumarokov A. M. Agro-association ‘Chysta Krynytsia’, 11, Tsentralna St., Drabynivka Village, Poltava District,

Poltava Region, 39351, UKRAINE; e-mail: sumarokov_sam@ukr.net, ORCID: 0000-0001-8115-851X

Zamoroka A. M. Vasyl Stefanyk Precarpathian National University, 57, Shevchenko St.,

Ivano-Frankivsk, 77018, UKRAINE; e-mail: andrew.zamoroka@pnu.edu.ua, ORCID: 0000-0001-5692-7997 
Eastern Carpathians except alpine meadows of the Chornohora Range and steppes in the Dnister River Valley. Coleoptera fauna is highly differentiated with a low similarity within habitats. We identified two different types of Coleoptera-mediated transmission of energy in meadow and forest habitats including the producer-consumer and the reducer-consumer energy transmission. We believe, this indicates on highest capacity and resilience of the forest ecosystems. Meanwhile, meadow ecosystems are more vulnerable to anthropogenic and natural threats. In light of our findings, there is a need to provide a special protection regime for Coleoptera habitats on alpine meadows and steppes in the region.

Keywords: altitudinal ecological gradient, $\beta$-diversity, functional diversity, ecosystem capacity. 4 figs, 2 tabs, 52 refs

I n t roduction. Coleoptera is the largest and the most diverse order not only within insects but also within all known groups of living organisms. There are over 350,000-400,000 described species of beetles, constituting about 25\% of all described species in the World (Stork et al., 2015; Audisio et al., 2015; Löbl, I., Smetana, 2007, 2010; Löbl, I., Löbl, D., 2015, 2016, 2017; Alonso-Zarazaga et al., 2017; Dariusz, Löbl, I., 2020). Some around 260,000 beetle species (65-75\% of all known Coleoptera) belong to the five families. These include Curculionidae $(\approx 83,000$ species $)$, Staphylinidae $(\approx 64,000$ species), Carabidae ( $\approx 43,000$ species), Chrysomelidae $(\approx 35,000$ species), and Cerambycidae ( $\approx 35,000$ species). In Europe, there are near 30,000 known species of beetles (Audisio et al., 2015; Löbl, I., Smetana, 2007, 2010; Löbl, I., Löbl, D., 2015, 2016, 2017; AlonsoZarazaga et al., 2017; Dariusz, Löbl, I., 2020). The exact number of beetles in Ukraine is unknown yet. We believe that there are at least 15,000 species in Ukraine.

The studies of Coleoptera fauna in the Eastern Carpathians have been started almost 200 years ago, in the third decade of the XIX century. Since that time, it is accumulated large data and formed sufficiently complete information on the local fauna of Coleoptera. However, a complete and exhaustive list of beetles has not yet been compiled for the region. The detailed historical revues of Coleoptera fauna studies are given in the multiple recent papers (Yanytsky, 2001; Rizun, 2003; Mateleshko, 2008; Zamoroka, 2009; Zamoroka et al., 2018).

The earliest known data on 17 species of beetles from the region was published in a shot note of A. Zawadzki (1825). However, detailed and comprehensive studies had been conducted only in the middle of the XIX century. M. Nowicki (1873) presented the first comprehensive list of Coleoptera from the region, which comprised 2,591 species. Decade later, M. Łomnicki (1884) listed 3,182 species of Coleoptera. At the beginning of the XX century, he listed 5,396 species of 76 families of Coleoptera for territory of Western Ukraine and Eastern Poland (Łomnicki, 1913). The important generalization for the understanding of the regional Coleoptera fauna was published in the monograph of R. Kuntze and J. Noskiewicz (1938), where authors provided the first analysis of the spatial distribution of Coleoptera fauna in the regional scale. They listed 181 species distributed exceptionally on Western Podillia and 126 species widespread outside of Western Podillia. V. Lazorko (1963) made a contribution to the knowledge of the regional fauna. He listed 323 species of Coleoptera, 21 of which were new for the region.

After World War II, the generalized coleopterological revues were not published. Instead, many authors specialized in deep studies of certain Coleoptera groups such as Buprestidae (Zahaykevych, 1978), Cerambycidae (Zahaykevych, 1959), Chrysomelidae (Brovdiy, 1977), Curculionidae (Tveritina, 1959), Elateridae (Dolin, 1966), Ipidae (Zahaykevych, 1957), Stahpylinidae (Petrenko, Nadvornyy, 1990), etc.

Modern research of Coleoptera focuses on faunistics, ecology, and phylogeography. The detailed faunistic studies of certain families of Coleoptera are presented in multiple papers. These include Carabidae (Rizun, 2003), Haliplidae, Noteridae, Dytiscidae, Gyrinidae, Hydraenidae, Hydrophilidae (Mateleshko, 2008), Staphylinidae (Petrenko, 2009), Pselaphidae (Krivosheyev, 2012), Cerambycidae (Zamoroka et al., 2012; Zamoroka, 2018), Buprestidae (Yanytsky, 2013), Curculionidae (Nikulina et al., 2015. Yunakov et al., 2018), Cryptophagidae (Ocheretna, 2020), etc. Ecological studies of Coleoptera include altitudinal distribution (Rizun, Tymochko, Chumak, 2004), habitat patterns (Rizun, Tymochko, Chumak, 2004; Zamoroka, 2008), the influence of anthropogenic impact (Chumak et al., 2005), local extinction (Zamoroka et al., 2018; Zamoroka, 2019), etc. The phylogeographic coleopterological studies focused on certain species (Kajtoch, Lachowska-Cierlik, Mazur, 2009; Kubisz et al., 2012; Zamoroka et al., 2019). Despite the sufficiently well-known local fauna of Coleoptera, a number of faunal, ecological and phylogeographic aspects need to be clarified especially in the light of current climatic changes, ecosystems restoration, and conservation.

In the current study, we presented the results of the study of 595 species of Coleoptera within protected areas in the Eastern Carpathians. Their spatial distribution and change under the influence of regional altitude gradients and differentiation within habitats.

Materials and methods. Region of study. The studied territory covers part of the Eastern Carpathian Mountains and its eastern foothills in Ivano-Frankivsk Region (Ukraine). There are four National Parks (NP) and one Nature Reserve (NR). These include Carpathian NP, Halych NP, Hutsulschyna NP, Verkhovyna NP, and Gorgany NR with a total area of $1,148.2 \mathrm{~km}^{2}$ or $8.2 \%$ of the region. The listed protected 
areas cover an altitudinal range from 203 to 2,061 m a. s. l. within the regional scale of variation of ecological conditions. Climatic conditions gradually vary from dry (average annual precipitation $600 \mathrm{~mm}$ ) and warm (average annual temperature $+7^{\circ} \mathrm{C}$ ) in the foothills $(200 \mathrm{ma}$. s. l.) to wet (average annual precipitation $2,500 \mathrm{~mm}$ ) and cold (average annual temperature $+4{ }^{\circ} \mathrm{C}$ ) in mountains highlands (2,000 m a. s. l.). Altitudinal zonation in the region includes steppes (200-350 m a. s. l.), oak-beech forests (300-400 m a. s. l.), oak-beech-fir forests (400-500 m a. s. l.), beech-fir forests (500-750 m a. s. 1.), beech-spruce-fir forests (650-900 m a. s. l.), firspruce forests $(900-1,050 \mathrm{ma}$ a. l.), pure spruce forests $(1,000-1,800 \mathrm{ma}$ a. l.), cedar pine-spruce forests (1,500-1,800 ma.s. l.), alpine shrubs (1,500-1,900 ma.s. l.), alpine meadows or stony placers (1,8002,061 m a. s. l.).

$\mathrm{H}$ a b i t a t s. We selected five main habitats (Table 1) within all altitudinal vegetation belts. These include steppe meadow, secondary fir-beech forest, primeval beech-fir forest, high mountain pasture meadow among the pure spruce forest, and alpine shrubs and meadows (Malynovskyi, Krichfalushiy, 2000; Hryhora, Solomakha, 2005).

Table 1. Location of the sample plots

\begin{tabular}{|l|l|c|c|l|}
\hline \multicolumn{1}{|c|}{ Locality } & \multicolumn{1}{|c|}{ Habitat } & $\begin{array}{c}\text { Decimal coordinates } \\
\text { (WGS84) }\end{array}$ & $\begin{array}{c}\text { Altitude, } \\
\text { m a. s. l. }\end{array}$ & \multicolumn{1}{|c|}{ Administrative location } \\
\hline Mezhyhirskyi Kamin & Steppe & $49.119281,24.804577$ & 325 & Mezhyhirtsi, Ivano-Frankivsk; NP Halych \\
\hline Zhenets & Secondary fir-beech forest & $48.373040,24.540655$ & 760 & Tatariv, Nadvirna; NP Carpathian \\
\hline Dzhurdzhiy & Primeval beech-fir forest & $48.478224,24.291243$ & 900 & Zelena, Nadvirna; NR Gorgany \\
\hline Vesnarka & High mountain meadows & $48.023633,24.658863$ & 1,450 & Zelene, Verkhovyna; NP Carpathian \\
\hline Pozhyzhevska & Alpine shrubs and meadows & $48.146276,24.524220$ & 1,800 & Vorokhta, Nadvirna; NP Carpathian \\
\hline
\end{tabular}

Plot 1. Mezhehirskyi Kamin is located in Halych NP (49.119281, 24.804577) within the westernmost margin of Podillia Upland. The locality occupies the elongated hill (0.85 km long), directed from north to south, with steep slopes and a flat top with numerous appearances of karst. Vegetation represents xerophilous steppe meadows with the dominating of Brachypodium pinnatum, Elytrigia intermedia, Festuca valesiaca, Carex humilis, Stipa capillata, Anthericum ramosum, Prunella grandiflora, Linum flavum, Chamaecytisus blockianus, Pyrethrum corymbosum, Inula ensifolia, Artemisia austriaca.

Plot 2. Zhenets is located in Carpathian NP (48.373040, 24.540655) in the Gorgany Range. It is a narrow valley of the Zhenets River (4.6 km long), directed to southeast. The valley is bordered by the Khomiak-Syniak Range (1,500-1,600 m a. s. l.) on south and the Yavirnyk Range (1,000-1,400 m a. s. l.) on north. Vegetation represents mountain fir-beech forests (up to $1,000 \mathrm{~m}$ a. s. 1.) and pure spruce forest (over $1000 \mathrm{~m}$ a. s. l.). Firbeech forests consist of Abies alba, Fagus sylvatica in the trees layer, and Oxalis acetosella, Carex pilosa, Dentaria glandulosa, Dentaria bulbifera, Anemone nemorosa, Athyrium filix-femina, Vaccinum myrtillus, Rubus hirtus in the herbs layer. Small patches of the secondary meadows are scattered among the valley.

Plot 3. Dzhurdzhiy is located in Gorgany NR (48.478224, 24.291243) in the Gorgany Range. It is a narrow valley of the Dzhurdzhynets River (3.8 km long), directed to north. The valley is bordered by the Berezovachka Range (1,200-1,500 m a. s. l.) on east and the Skalky Range (1,300-1,500 m a. s. l.) on west. Vegetation represents primeval mountain beech-fir forests (up to 1,000 m a. s. 1.) and pure spruce forest and cedar pine-spruce forests (over 1,000 ma.s. 1.). Beech-fir forests consist of Abies alba, Fagus sylvatica, Acer pseudoplatanus in the trees layer, and Lonicera nigra, Sambucus racemosa, Sorbus aucuparia in the shrub layer, and Vaccinum myrtillus, Oxalis acetosella, Lusula sylvatica, Dryopteris affinis, Carex pilosa, Dentaria glandulosa, Soldanella hungarica in the herbs layer.

Plot 4. Vesnarka is located in Carpathian NP $(48.023633,24.658863)$ in the Chornohora Range. It is a high mountain pasture meadow (local name 'polonyna') on the mountain range of mount Pip Ivan Chornohirskyi. The location is surrounded by pure spruce forest. Vegetation of the polonyna is represented by dominating Deschampsia caespitosa, Phleum alpinum, Luzula luzuloides, Festuca picturata, Festuca rubra, Potentilla aurea, Ligusticum mutellina, Rumex alpinum. Pure spruce forests consist of Picea abies at trees level and Oxalis acetosella, Euphorbia amygdaloides, Dentaria glandulosa, Dentaria bulbifera, Anemone nemorosa in the herbs layer.

Plot 5. Pozhyzhevska is located in Carpathian NP (48.146276, 24.524220) in the Chornohora Range. It is an alpine meadow with patches of alpine shrubs around of summit of mount Pozhyzhevska. The vegetation of the alpine meadows is represented by dominating Carex sempervirens, Juncus trifidus, Sesleria coerulans, Calamagrostis villosa, Phleum alpinum, Festuca supina, Rhododendron myrtifolium, Potentilla aurea, Soldanella hungarica. Vegetation of the alpine shrubs consists Pinus mugo, Alnus viridis in shrubs layer, and Rhododendron myrtifolium, Vaccinum myrtillus, Empetrum nigrum, Athyrium filix-femina, Deschampsia caespitosa, Luzula 
sylvatica, Nardus stricta, Doronicum pardalianches, Senecio fuchsii in herbs layer, and Sphagnum magellanicum in moss layer.

Collecting methods. Materials were collected during 2010-2013. The multiple methods of material collecting were applied. These included manual collecting insects on the forage plants, on withered, felled, and damaged broadleaf and coniferous trees, beating tray, flotation method, using entomological sweepnet, pitfall traps (line of 10 cans with an interval of $5 \mathrm{~m}$ ), and light traps (combining of day-light and UV-light lamps) (Ghilarov, 1941; Fasulati, 1971; Grootaert et al., 2010; Steiner, Häuser, 2010). We also studied additional materials collected during 1993-1996 (A. Sumarokov's collection).

Insects sampling was carried under the limits set by the Ministry of Ecology and Natural Resources of Ukraine for Carpathian NP (2011-2013), Halych NP (2010-2011), and Gorgany NR (2013). All material has been collected under approved ethics guidelines (Drinkwater, Robinson, Hard, 2019; Fischer, Larson, 2019).

Taxonomy and nomenclature. In giving species names and taxonomy, we followed 'Catalogue of Palaearctic Coleoptera’ (Löbl, I., Smetana, 2007, 2010; Löbl, I., Löbl, D., 2015, 2016, 2017; Dariusz, Löbl, I., 2020) and 'Cooperative Catalogue of Palaerctic Coleoptera Curculionoidea' (Alonso-Zarazaga et al., 2017).

Results and discussions. General diversity patterns. Comprehensive collection methods allowed us to identify 595 beetle species of 37 families (Table 2) on the territory of protected areas of the Eastern Carpathians. We found 344 species in Carpathian NP, 327 species - in Halych NP, and 192 species - in Gorgany NR. These include Carabidae (169 species), Curculionidae (107 species), Chrysomelidae (59 species), Cerambycidae (49 species), Staphylinidae (30 species), Cantharidae (22 species), Elateridae (22 species), Coccinellidae (15 species), Brentidae (14 species), Silphidae (11 species), Attelabidae (10 species), Melyridae (10 species), Scarabaeidae (10 species). Remained 24 families consist of less than 10 species. Taxonomical composition (Fig. 1) of Coleoptera fauna highly varies among studied territory. While the number of Coleoptera families decreases evenly along with increasing in altitude, the proportion per family dramatically varies within the studied area. For instance, we found 26 families at an altitude $325 \mathrm{~m}$ a. s. 1 . in Halych NP, 27 families - at $760 \mathrm{~m}$ a. s. l. in Carpathian NP, 25 families - at $900 \mathrm{~m} \mathrm{a.} \mathrm{s.} \mathrm{1.} \mathrm{in} \mathrm{Gorgany} \mathrm{NR,}$ 19 families - at 1,450 m a. s. l. in Carpathian NP, and 15 families — at 1,800 m a. s. l. in Carpathian NP.

Tab le 2. Taxonomic composition and spatial distribution of Coleoptera among the sample plots

\begin{tabular}{|c|c|c|c|c|c|}
\hline Species & Mezhyhirskyi Kamin & Zhenets & Dzhurdzhiy & Vesnarka & Pozhyzhevska \\
\hline \multicolumn{6}{|l|}{ ATTELABIDAE } \\
\hline Apoderus coryli (Linnaeus, 1758) & $*$ & - & - & - & - \\
\hline Attelabus nitens (Scopoli, 1763) & - & $*$ & - & - & - \\
\hline Byctiscus betulae (Linnaeus, 1758) & - & - & - & - & $*$ \\
\hline Byctiscus populi (Linnaeus, 1758) & $*$ & - & - & - & - \\
\hline Deporaus betulae (Linnaeus, 1758) & $*$ & - & - & - & - \\
\hline Involvulus cupreus (Linnaeus, 1761) & $*$ & - & - & - & - \\
\hline Involvulus pubescens (Fabricius, 1775) & * & - & - & - & - \\
\hline Mecorhis aethiops (Bach, 1854) & $*$ & - & - & - & - \\
\hline Neocoenorrhinus germanicus (Herbst, 1797) & $*$ & $*$ & - & - & - \\
\hline Tatianaerhynchites aequatus (Linnaeus, 1767) & $*$ & - & - & - & - \\
\hline Subtotal: & 8 & 2 & 0 & 0 & 1 \\
\hline \multicolumn{6}{|l|}{ BRENTIDAE } \\
\hline Apion cruentatum Walton, 1844 & - & $*$ & - & - & - \\
\hline Apion frumentarium (Linnaeus, 1758) & $*$ & - & - & - & - \\
\hline Cyanapion gyllenhalii (W. Kirby, 1808) & $*$ & - & - & - & - \\
\hline Eutrichapion viciae (Paykull, 1800) & $*$ & - & - & - & - \\
\hline Holotrichapion pisi (Fabricius, 1801) & - & $*$ & - & - & - \\
\hline Ischnopterapion loti (W. Kirby, 1808) & $*$ & & - & - & - \\
\hline Nanophyes marmoratus (Goeze, 1777) & - & $*$ & - & - & - \\
\hline Oxystoma ochropus (Germar, 1818) & - & $*$ & - & - & - \\
\hline Perapion violaceum (W. Kirby, 1808) & $*$ & - & - & - & - \\
\hline Protapion apricans (Herbst, 1797) & $*$ & $*$ & - & - & - \\
\hline Protapion assimile (Kirby, 1808) & $*$ & & - & - & - \\
\hline Protapion fulvipes (Geoffroy, 1785) & - & $*$ & $*$ & - & - \\
\hline Protapion gracilipes (Dietrich, 1857) & $*$ & - & - & - & - \\
\hline Pseudostenapion simum (Germar, 1817) & $*$ & - & - & - & - \\
\hline Subtotal: & 9 & 6 & 1 & 0 & 0 \\
\hline \multicolumn{6}{|l|}{ BUPRESTIDAE } \\
\hline Anthaxia helvetica Stierlin, 1868 & - & * & $*$ & - & - \\
\hline
\end{tabular}


A. M. SUMAROKOV, A. M. ZAMOROKA

Spatial patterns and habitat differentiation of the beetle (Insecta: Coleoptera) fauna of protected areas in the Eastern Carpathians (Ivano-Frankivsk Region, Ukraine)

Continuation of Table 2

\begin{tabular}{|c|c|c|c|c|c|}
\hline Species & Mezhyhirskyi Kamin & Zhenets & Dzhurdzhiy & Vesnarka & Pozhyzhevska \\
\hline Anthaxia nitidula signaticollis (Krynicki, 1832) & - & - & - & - & $*$ \\
\hline Coraebus elatus (Fabricius, 1787) & * & - & - & - & - \\
\hline Trachys minutus minutus (Linnaeus, 1758) & - & - & - & - & $*$ \\
\hline Subtotal: & 1 & 1 & 1 & 0 & 2 \\
\hline \multicolumn{6}{|l|}{ BYRRHIDAE } \\
\hline Byrrhus pilula (Linnaeus, 1758) & - & - & $*$ & - & - \\
\hline Byrrhus glabratus Heer, 1841 & - & $*$ & $*$ & - & - \\
\hline Byrrhus luniger Germar, 1817 & - & $*$ & $*$ & - & $*$ \\
\hline Carpathobyrrhulus transsylvanicus (Suffrian, 1848) & - & - & $*$ & - & - \\
\hline Cytilus sericeus (Forster, 1771) & - & $*$ & $*$ & - & - \\
\hline Subtotal: & 0 & 3 & 5 & 0 & 1 \\
\hline \multicolumn{6}{|l|}{ BYTURIDAE } \\
\hline Byturus ochraceus (Scriba, 1790) & $*$ & $*$ & - & $*$ & - \\
\hline Byturus tomentosus (De Geer, 1774) & - & $*$ & - & - & $*$ \\
\hline Subtotal: & 1 & 2 & 0 & 1 & 1 \\
\hline \multicolumn{6}{|l|}{ CANTHARIDAE } \\
\hline Ancistronycha abdominalis (Fabricius, 1798) & - & $*$ & - & - & - \\
\hline Ancistronycha violacea (Paykull, 1798) & - & $*$ & - & - & - \\
\hline Cantharis annularis Ménétriés, 1836 & - & $* *$ & $* *$ & - & - \\
\hline Cantharis flavilabris Fallén, 1807 & - & $*$ & - & - & - \\
\hline Cantharis fusca Linnaeus, 1758 & $*$ & $*$ & $*$ & - & - \\
\hline Cantharis livida var. rufipes Herbst, 1784 & $* *$ & - & $* *$ & - & - \\
\hline Cantharis nigricans (O. F. Müller, 1776) & $*$ & $* *$ & $*$ & - & - \\
\hline Cantharis obscura Linnaeus, 1758 & $* * *$ & $*$ & $*$ & - & - \\
\hline Cantharis pellucida Fabricius, 1792 & $*$ & $*$ & $*$ & - & - \\
\hline Cantharis quadripunctata (O. F. Müller, 1776) & - & $*$ & $*$ & - & - \\
\hline Cantharis rufa Linnaeus, 1758 & $* *$ & $*$ & $*$ & $*$ & - \\
\hline Cantharis rustica Fallén, 1807 & $*$ & $* *$ & $*$ & - & - \\
\hline Malthodes sp. & - & - & $*$ & - & - \\
\hline Metacantharis discoidea (Ahrens, 1812) & - & $*$ & - & - & - \\
\hline Podabrus alpinus (Paykull, 1798) & - & * & - & - & - \\
\hline Podistra rufotestacea (Letzner, 1845) & - & $*$ & - & - & - \\
\hline Podistra schoenherri (Dejean, 1837) & - & $*$ & - & - & - \\
\hline Rhagonycha fulva (Scopoli, 1763) & $* * *$ & $*$ & - & - & - \\
\hline Rhagonycha lignosa (O. F. Müller, 1764) & - & $*$ & ** & - & - \\
\hline Rhagonycha nigriventris Motschulsky, 1860 & $*$ & $*$ & $*$ & $*$ & - \\
\hline Rhagonycha testacea (Linnaeus, 1758) & - & * & * & - & - \\
\hline Silis nitidula Fabricius, 1792 & $*$ & - & - & - & - \\
\hline Subtotal: & 10 & 19 & 13 & 2 & 0 \\
\hline \multicolumn{6}{|l|}{ CARABIDAE } \\
\hline Abax parallelepipedus (Piller et Mitterpacher, 1783) & * & $* * *$ & $* *$ & $*$ & - \\
\hline Agonum fuliginosum (Panzer, 1809) & - & $*$ & - & - & - \\
\hline Agonum muelleri (Herbst, 1784) & * & - & $*$ & - & - \\
\hline Agonum sexpunctatum (Linnaeus, 1758) & - & $*$ & - & - & - \\
\hline Agonum versutum Sturm, 1824 & $*$ & - & $*$ & - & - \\
\hline Agonum viduum (Panzer 1796) & - & $*$ & $*$ & - & - \\
\hline Agonum viridicupreum (Goeze, 1777) & - & $*$ & - & - & - \\
\hline Amara aenea (De Geer, 1774) & $* *$ & - & - & - & - \\
\hline Amara bifrons (Gyllenhal, 1810) & - & $*$ & - & - & - \\
\hline Amara communis (Panzer 1797) & - & $*$ & $*$ & - & - \\
\hline Amara consularis (Duftschmid, 1812) & $*$ & - & $*$ & - & - \\
\hline Amara convexior Stephens, 1828 & - & - & $*$ & - & - \\
\hline Amara curta Dejean, 1828 & - & - & $*$ & - & - \\
\hline Amara eurynota (Panzer, 1796) & - & $*$ & - & - & - \\
\hline Amara familiaris (Duftschmid, 1812) & $*$ & - & - & - & - \\
\hline Amara ovata (Fabricius, 1792) & $*$ & - & $*$ & - & - \\
\hline Amara plebeja (Gyllenhal, 1810) & * & $*$ & - & - & - \\
\hline Amara similata (Gyllenhal, 1810) & $*$ & - & - & - & - \\
\hline Anchomenus dorsalis (Pontoppidan, 1763) & - & $*$ & - & - & - \\
\hline Anisodactylus binotatus (Fabricius, 1787) & $*$ & $*$ & - & - & - \\
\hline Anisodactylus nemorivagus (Duftschmid, 1812) & - & $*$ & - & - & - \\
\hline Anisodactylus signatus (Panzer, 1796) & - & - & $*$ & - & - \\
\hline Asaphidion caraboides (Schrank, 1781) & - & $*$ & $*$ & - & - \\
\hline
\end{tabular}


Continuation of Table 2

\begin{tabular}{|c|c|c|c|c|c|}
\hline $\begin{array}{r}\text { Species } \\
\end{array}$ & Mezhyhirskyi Kamin & Zhenets & Dzhurdzhiy & Vesnarka & Pozhyzhevska \\
\hline Asaphidion flavipes (Linnaeus, 1761) & $\left.\right|^{*}$ & - & $*$ & - & - \\
\hline Badister bullatus (Schrank, 1798) & * & - & $*$ & - & - \\
\hline Badister lacertosus Sturm, 1815 & - & $*$ & - & - & - \\
\hline Badister peltatus (Panzer, 1796) & $*$ & - & - & - & - \\
\hline Bembidion argenteolum Ahrens, 1812 & ** & - & - & - & - \\
\hline Bembidion articulatum (Panzer, 1796) & - & - & $*$ & - & - \\
\hline Bembidion ascendens K. Daniel, 1902 & $*$ & $*$ & - & - & - \\
\hline Bembidion assimile Gyllenhal, 1810 & - & $* *$ & - & - & - \\
\hline Bembidion atrocoeruleum (Stephens, 1828) & - & - & $*$ & - & - \\
\hline Bembidion bipunctatum nivale Heer, 1837 & $* *$ & - & $*$ & - & - \\
\hline Bembidion conforme Dejean, 1831 & $*$ & - & - & - & - \\
\hline Bembidion dalmatinum Dejean, 1831 & - & $*$ & $*$ & - & - \\
\hline Bembidion dentellum (Thunberg, 1787) & - & $*$ & - & - & - \\
\hline Bembidion doderoi Ganglbauer, 1891 & - & - & $*$ & - & - \\
\hline Bembidion doris (Panzer, 1796) & - & $*$ & - & - & - \\
\hline Bembidion fasciolatum (Duftschmid, 1812) & - & $* *$ & - & - & - \\
\hline Bembidion geniculatum Heer, 1837 & $* *$ & - & $*$ & - & $* *$ \\
\hline Bembidion guttula (Fabricius, 1792) & $* *$ & $* *$ & - & - & - \\
\hline Bembidion lampros (Herbst, 1784) & ** & $* *$ & $*$ & - & - \\
\hline Bembidion litorale (Oliver, 1790) & $*$ & - & $*$ & - & - \\
\hline Bembidion lunatum (Duftschmid, 1812) & $*$ & $* *$ & $*$ & - & - \\
\hline Bembidion lunulatum (Geoffroy, 1785) & - & $*$ & - & - & - \\
\hline Bembidion mannerheimii C. R. Sahlberg, 1827 & $*$ & $*$ & $*$ & - & - \\
\hline Bembidion milleri Jacquelin du Val, 1852 & - & - & - & - & $*$ \\
\hline Bembidion monticola monticola Sturm, 1825 & - & $* *$ & $*$ & - & - \\
\hline Bembidion nigricorne Gyllenhal, 1827 & - & - & - & - & * \\
\hline Bembidion properans (Stephens, 1828) & ** & $* *$ & - & - & - \\
\hline Bembidion pygmaeum (Fabricius, 1792) & - & $*$ & - & - & - \\
\hline Bembidion quadrimaculatum (Linnaeus, 1761) & $*$ & ** & $*$ & - & - \\
\hline Bembidion quadripustulatum Audinet-Serville, 1821 & $*$ & $*$ & - & - & - \\
\hline Bembidion ruficorne Sturm, 1825 & - & $* *$ & $*$ & - & - \\
\hline Bembidion saxatile saxatile Gyllenhal, 1827 & - & - & $*$ & - & - \\
\hline Bembidion scapulare Dejean, 1831 & - & - & $*$ & - & - \\
\hline Bembidion schueppeli Dejean, 1831 & $* *$ & $* *$ & $*$ & - & - \\
\hline Bembidion splendidum Sturm, 1825 & - & $* *$ & - & - & - \\
\hline Bembidion striatum (Fabricius, 1792) & $* *$ & $*$ & - & - & - \\
\hline Bembidion tetracolum Say, 1823 & $* *$ & * & $*$ & - & - \\
\hline Bembidion tibiale (Duftschmid, 1812) & - & $*$ & $*$ & - & - \\
\hline Bembidion varicolor (Fabricius, 1803) & - & - & $*$ & - & - \\
\hline Bembidion varium (Olivier, 1795) & - & $* *$ & $*$ & - & - \\
\hline Bembidion velox (Linnaeus, 1761) & - & $* *$ & - & - & - \\
\hline Calathus fuscipes (Goeze, 1777) & $*$ & $*$ & $*$ & - & - \\
\hline Calathus melanocephalus (Linnaeus, 1758) & $*$ & $* *$ & $*$ & - & - \\
\hline Calathus metallicus Dejean, 1828 & - & - & $* * *$ & - & - \\
\hline Carabus arcensis emerita Fischer von Waldheim, 1823 & - & - & - & - & $*$ \\
\hline Carabus auronitens escheri Palliardi, 1825 & - & $* * *$ & $* * *$ & * & * \\
\hline Carabus cancellatus cancellatus Illiger, 1798 & $*$ & $* *$ & $*$ & - & - \\
\hline Carabus convexus convexus Fabricius, 1775 & $*$ & - & $*$ & - & - \\
\hline Carabus coriaceus rugifer (Kraatz, 1877) & $*$ & $* *$ & $* *$ & - & - \\
\hline Carabus excellens Fabricius, 1798 & $*$ & - & - & - & - \\
\hline Carabus fabricii ucrainicus Lazorko, 1951 & - & - & $*$ & - & - \\
\hline Carabus glabratus glabratus Paykull, 1790 & $*$ & $* *$ & $*$ & * & $*$ \\
\hline Carabus granulatus granulatus Linnaeus, 1758 & $*$ & $* *$ & $*$ & - & - \\
\hline Carabus intricatus Linnaeus, 1761 & $*$ & - & - & - & - \\
\hline Carabus irregularis montandoni Buysson, 1882 & - & $*$ & - & - & - \\
\hline Carabus linnei Panzer, 1810 & - & $* *$ & $* *$ & $*$ & - \\
\hline Carabus obsoletus Sturm, 1815 & - & - & ** & * & * \\
\hline Carabus rothi hampei Küster, 1846 & - & - & - & - & * \\
\hline Carabus scheidleri zawadzkii Kraatz, 1854 & - & $* * *$ & $* * *$ & * & $*$ \\
\hline Carabus sylvestris transsylvanicus Dejean, 1826 & - & - & - & $*$ & $* * *$ \\
\hline Carabus variolosus Fabricius, 1787 & - & $* *$ & $*$ & - & - \\
\hline Carabus violaceus Linnaeus, 1758 & $*$ & $* * *$ & $* *$ & $*$ & - \\
\hline Chlaenius spoliatus spoliatus (P. Rossi, 1792) & - & - & $*$ & - & - \\
\hline Chlaenius vestitus (Paykull, 1790) & - & - & $*$ & - & - \\
\hline
\end{tabular}


A. M. SUMAROKOV, A. M. ZAMOROKA Spatial patterns and habitat differentiation of the beetle (Insecta: Coleoptera) fauna of protected areas in the Eastern Carpathians (Ivano-Frankivsk Region, Ukraine)

Continuation of Table 2

\begin{tabular}{r|}
\hline Species \\
\hline Cicindela campestris Linnaeus, 1758 \\
\hline Cicindela sylvicola Dejent
\end{tabular}

Cicindela sylvicola Dejean, 1822

Clivina collaris (Herbst, 1784)

Clivina fossor (Linnaeus, 1758)

Cychrus caraboides (Linnaeus, 1758)

Cylindera germanica (Linnaeus, 1758)

Cymindis cingulata Dejean 1825

Cymindis humeralis (Geoffroy, 1785)

Deltomerus carpathicus (L. Miller, 1868)

Dicheirotrichus obsoletus (Dejean, 1829)

Drypta dentata (P. Rossi, 1790)

Duvalius subterraneus (L. Miller, 1868)

Dyschirius aeneus (Dejean, 1825)

Dyschirius globosus (Herbst, 1784)

Dyschirius rufipes (Dejean, 1825)

Elaphrus aureus P. W. J. Müller, 1821

Elaphrus cupreus Duftschmid, 1812

Elaphrus riparius (Linnaeus, 1758)

Harpalus affinis (Schrank, 1781)

Harpalus calceatus (Duftschmid, 1812)

Harpalus distinguendus (Duftschmid, 1812)

Harpalus griseus (Panzer, 1796)

Harpalus latus (Linnaeus, 1758)

Harpalus rubripes (Duftschmid, 1812)

Harpalus serripes (Quensel in Schönherr, 1806)

Harpalus smaragdinus (Duftschmid, 1812)

Harpalus tardus (Panzer, 1797)

Harpalus rufipes (De Geer, 1774)

Laemostenus terricola (Herbst, 1784)

Lebia cruxminor (Linnaeus, 1758)

Lebia cyanocephala (Linnaeus, 1758)

Leistus ferrugineus (Linnaeus, 1758)

Leistus piceus (Frölich, 1799)

Licinus depressus (Paykull, 1790)

Licinus hoffmannseggii (Panzer, 1803)

Microlestes maurus (Sturm, 1827)

Microlestes plagiatus (Duftschmid, 1812)

Molops piceus (Panzer, 1793)

Nebria brevicollis (Fabricius, 1792)

Nebria fuscipes Fuss, 1849

Nebria jockischii hoepfneri Dejean, 1826

Nebria picicornis (Fabricius, 1801)

Nebria reitteri Rybiński, 1902

Nebria rufescens rufescens (Ström, 1768)

Nebria transsylvanica Germar 1824

Notiophilus aquaticus (Linnaeus, 1758)

Notiophilus biguttatus (Fabricius, 1779)

Notiophilus palustris (Duftschmid, 1812)

Notiophilus rufipes Curtis, 1829

Oodes helopioides (Fabricius, 1792)

Ophonus rufibarbis (Fabricius, 1792)

Ophonus rupicola (Sturm, 1818)

Oxypselaphus obscurus (Herbst, 1784)

Panagaeus bipustulatus (Fabricius, 1775)

Panagaeus cruxmajor (Linnaeus, 1758)

Patrobus quadricollis L. Miller, 1868

Perileptus areolatus (Creutzer, 1799)

Platynus assimilis (Paykull, 1790)

Poecilus lepidus (Leske, 1785)

Poecilus versicolor (Sturm, 1824)

Pterostichus anthracinus (Illiger, 1798)

Pterostichus diligens (Sturm, 1824)

Pterostichus foveolatus (Duftschmid, 1812)

Pterostichus jurinei (Panzer, 1803) \begin{tabular}{|c|c|c|c|c|}
\hline Mezhyhirskyi Kamin & Zhenets & Dzhurdzhiy & Vesnarka & Pozhyzhevska \\
\hline$* * *$ & $*$ & $*$ & - & - \\
\hline
\end{tabular}

\begin{tabular}{|c|c|c|c|c|}
\hline & & & - & \\
\hline- & - & $* * *$ & - & - \\
\hline$*$ & - & $*$ & - & - \\
\hline$* *$ & * & $*$ & - & - \\
\hline$*$ & $*$ & $* *$ & $*$ & $* * *$ \\
\hline$* *$ & - & - & - & - \\
\hline- & - & $*$ & - & - \\
\hline$*$ & - & $*$ & - & - \\
\hline- & $* * *$ & $*$ & - & - \\
\hline- & - & $*$ & - & - \\
\hline$* *$ & $*$ & - & - & - \\
\hline- & - & $*$ & - & - \\
\hline- & * & - & - & - \\
\hline- & $*$ & $*$ & - & - \\
\hline$*$ & - & - & - & - \\
\hline- & - & $*$ & - & - \\
\hline- & $*$ & $*$ & - & - \\
\hline- & $*$ & - & - & - \\
\hline$*$ & - & $*$ & - & - \\
\hline$* * *$ & $*$ & - & - & - \\
\hline- & $* *$ & - & - & - \\
\hline- & $*$ & $*$ & - & - \\
\hline$* *$ & $*$ & - & - & - \\
\hline$* *$ & $*$ & - & - & - \\
\hline- & $* *$ & - & - & - \\
\hline- & $*$ & - & - & - \\
\hline$*$ & - & - & - & - \\
\hline$*$ & $*$ & $*$ & - & - \\
\hline- & - & $*$ & - & - \\
\hline- & - & $*$ & - & - \\
\hline- & $*$ & - & - & - \\
\hline$* * *$ & - & - & - & - \\
\hline- & * & $*$ & - & - \\
\hline$*$ & - & $*$ & - & - \\
\hline- & $*$ & - & - & - \\
\hline$*$ & * & - & - & - \\
\hline- & $*$ & - & - & - \\
\hline- & $* *$ & $*$ & $*$ & - \\
\hline- & $*$ & $*$ & - & - \\
\hline- & - & $*$ & - & - \\
\hline- & - & $*$ & - & - \\
\hline- & - & $*$ & - & - \\
\hline- & - & $*$ & - & $*$ \\
\hline- & $*$ & - & - & - \\
\hline- & - & $*$ & - & $* * *$ \\
\hline- & - & * & - & - \\
\hline$*$ & * & $*$ & - & - \\
\hline$*$ & - & $*$ & - & $*$ \\
\hline$*$ & $*$ & - & - & - \\
\hline * & * & - & - & - \\
\hline$*$ & - & - & - & - \\
\hline$* * *$ & - & - & - & - \\
\hline$*$ & $*$ & - & - & - \\
\hline$*$ & - & - & - & - \\
\hline- & - & $*$ & - & - \\
\hline- & $*$ & $*$ & $*$ & - \\
\hline- & $*$ & $*$ & - & - \\
\hline- & $* * *$ & $* * *$ & - & - \\
\hline- & - & $*$ & - & - \\
\hline$*$ & - & $*$ & - & - \\
\hline$*$ & $*$ & $*$ & - & - \\
\hline$*$ & - & $*$ & - & - \\
\hline- & $* * *$ & $* *$ & - & $*$ \\
\hline- & $*$ & $*$ & - & $*$ \\
\hline
\end{tabular}


Continuation of Table 2

\begin{tabular}{|c|c|c|c|c|c|}
\hline Species & Mezhyhirskyi Kamin & Zhenets & Dzhurdzhiy & Vesnarka & Pozhyzhevska \\
\hline Pterostichus melanarius (Illiger, 1798) & $*$ & $*$ & $* *$ & - & - \\
\hline Pterostichus niger (Schaller, 1783) & $*$ & $*$ & $*$ & - & - \\
\hline Pterostichus nigrita (Paykull, 1790) & $*$ & $*$ & $*$ & - & - \\
\hline Pterostichus oblongopunctatus (Fabricius, 1787) & $*$ & - & $* *$ & - & $*$ \\
\hline Pterostichus ovoideus (Sturm, 1824) & - & $*$ & $*$ & - & - \\
\hline Pterostichus pilosus pilosus (Host, 1789) & - & - & $* * *$ & - & $*$ \\
\hline Pterostichus strenuus (Panzer, 1796) & $*$ & $*$ & $*$ & - & - \\
\hline Pterostichus unctulatus (Duftschmid, 1812) & - & $* *$ & - & - & * \\
\hline Pterostichus vernalis (Panzer, 1796) & ** & $*$ & $*$ & - & - \\
\hline Stenolophus teutonus (Schrank, 1781) & - & - & $*$ & - & - \\
\hline Synuchus vivalis (Illiger, 1798) & - & - & - & - & $*$ \\
\hline Tachys micros (Fischer von Waldheim, 1828) & - & $*$ & $*$ & - & - \\
\hline Trechus carpaticus Rybiński, 1902 & - & - & $*$ & - & - \\
\hline Trechus latus Putzeys, 1847 & - & - & - & - & $*$ \\
\hline Trechus pulchellus Putzeys, 1846 & - & - & $*$ & - & - \\
\hline Trechus quadristriatus (Schrank, 1781) & $* *$ & $*$ & - & - & - \\
\hline Trichotichnus laevicollis (Duftschmid, 1812) & - & $*$ & - & - & - \\
\hline Zabrus tenebrioides (Goeze, 1777) & $*$ & $*$ & - & - & - \\
\hline Subtotal: & 75 & 96 & 102 & 11 & 20 \\
\hline \multicolumn{6}{|l|}{ CERAMBYCIDAE } \\
\hline Agapanthia cardui (Linnaeus, 1767) & $* * *$ & - & - & - & - \\
\hline Agapanthia intermedia Ganglbauer, 1884 & $* *$ & - & - & - & - \\
\hline Agapanthia villosoviridescens (De Geer, 1775) & ** & - & - & $*$ & - \\
\hline Alosterna tabacicolor (De Geer, 1775) & $*$ & $* * *$ & $* * *$ & - & - \\
\hline Anaglyptus mysticus (Linnaeus, 1758) & $* *$ & $*$ & - & - & - \\
\hline Anastrangalia dubia (Scopoli, 1763) & - & $* * *$ & $* * *$ & $* *$ & - \\
\hline Anastrangalia sanguinolenta (Linnaeus, 1761) & - & $* *$ & $* *$ & $*$ & $*$ \\
\hline Anoplodera sexguttata (Fabricius, 1775) & $* *$ & - & - & - & - \\
\hline Calamobius filum (Rossi, 1790) & $* * *$ & - & & - & - \\
\hline Cyrtoclytus capra (Germar, 1824) & - & $*$ & $*$ & - & - \\
\hline Dinoptera collaris (Linnaeus, 1758) & $* *$ & $* * *$ & $* * *$ & - & - \\
\hline Dorcadion fulvum opillicum Zamoroka, 2019 & $* * *$ & - & - & - & - \\
\hline Dorcadion holosericeum Krynicki, 1832 & $* * *$ & - & - & - & - \\
\hline Etorofus pubescens (Fabricius, 1787) & - & - & - & $*$ & - \\
\hline Evodinus clathratus (Fabricius, 1793) & - & $* * *$ & $* * *$ & *** & ** \\
\hline Gaurotes virginea (Linnaeus, 1758) & - & $* * *$ & $* * *$ & - & - \\
\hline Hylotrupes bajulus (Linnaeus, 1758) & - & $*$ & - & - & - \\
\hline Judolia sexmaculata (Linnaeus, 1758) & - & $* *$ & $*$ & - & - \\
\hline Leiopus nebulosus (Linnaeus, 1758) & $*$ & - & - & - & - \\
\hline Leptura annularis Fabricius, 1801 & $* *$ & $*$ & $*$ & $*$ & - \\
\hline Leptura quadrifasciata Linnaeus, 1758 & - & $* * *$ & $* * *$ & $* *$ & - \\
\hline Lepturobosca virens (Linnaeus, 1758) & - & $* *$ & $* * *$ & $* *$ & - \\
\hline Molorchus minor (Linnaeus, 1758) & - & $* * *$ & $* * *$ & - & - \\
\hline Monochamus sartor (Fabricius, 1787) & - & $* * *$ & $* * *$ & $* * *$ & - \\
\hline Monochamus sutor (Linnaeus, 1758) & - & $* * *$ & $* * *$ & $* * *$ & - \\
\hline Nivellia sanguinosa (Gyllenhal, 1827) & - & $*$ & $* *$ & $* * *$ & - \\
\hline Oberea erythrocephala (Schrank, 1776) & $* *$ & - & - & - & - \\
\hline Obrium brunneum (Fabricius, 1792) & - & $* * *$ & - & - & - \\
\hline Oxymirus cursor (Linnaeus, 1758) & - & - & - & $* * *$ & $* *$ \\
\hline Pachyta quadrimaculata (Linnaeus, 1758) & - & $* *$ & - & $*$ & - \\
\hline Pachytodes cerambyciformis (Schrank, 1781) & $*$ & $* * *$ & $*$ & - & - \\
\hline Paracorymbia maculicornis (De Geer, 1775) & $*$ & - & $*$ & - & - \\
\hline Phytoecia affinis (Harrer, 1784) & $*$ & $*$ & $*$ & - & - \\
\hline Phytoecia coerulescens (Scopoli, 1763) & $* * *$ & - & - & - & - \\
\hline Phytoecia cylindrica (Linnaeus, 1758) & $*$ & $*$ & - & - & - \\
\hline Phytoecia tigrina Mulsant, 1851 & $*$ & - & - & - & - \\
\hline Phytoecia uncinata (W. Redtenbacher, 1842) & $* *$ & - & - & - & - \\
\hline Pidonia lurida (Fabricius, 1793) & - & $* * *$ & $* * *$ & - & - \\
\hline Pseudovadonia livida (Fabricius, 1776) & - & $*$ & - & - & - \\
\hline Rhagium inquisitor (Linnaeus, 1758) & - & $* * *$ & $* * *$ & $* * *$ & - \\
\hline Rhagium mordax (De Geer, 1775) & $*$ & - & $*$ & - & - \\
\hline Rutpela maculata (Poda, 1761) & $* *$ & - & - & - & - \\
\hline Stenocorus meridianus (Linnaeus, 1758) & - & $*$ & - & - & - \\
\hline Stenostola ferrea (Schrank, 1776) & $*$ & - & - & - & - \\
\hline
\end{tabular}


A. M. SUMAROKOV, A. M. ZAMOROKA Spatial patterns and habitat differentiation of the beetle (Insecta: Coleoptera) fauna of protected areas in the Eastern Carpathians (Ivano-Frankivsk Region, Ukraine)

Continuation of Table 2

\begin{tabular}{|c|c|c|c|c|c|}
\hline Species & Mezhyhirskyi Kamin & Zhenets & Dzhurdzhiy & Vesnarka & Pozhyzhevska \\
\hline Stenurella melanura (Linnaeus, 1758) & - & $* * *$ & - & - & - \\
\hline Stenurella nigra (Linnaeus, 1758) & $* *$ & - & - & - & - \\
\hline Stictoleptura rubra (Linnaeus, 1758) & - & $* * *$ & $* * *$ & $* * *$ & - \\
\hline Strangalia attenuata (Linnaeus, 1758) & $* * *$ & - & - & - & - \\
\hline Tetropium castaneum (Linnaeus, 1758) & - & $* * *$ & $* * *$ & $* * *$ & - \\
\hline Subtotal: & 25 & 29 & 23 & 16 & 3 \\
\hline \multicolumn{6}{|l|}{ CHRYSOMELIDAE } \\
\hline Agelastica alni (Linnaeus, 1758) & $*$ & $* * *$ & - & - & - \\
\hline Aulacophora quadrimaculata (Fabricius, 1781) & $* *$ & - & - & - & - \\
\hline Bromius obscurus (Linnaeus, 1758) & - & - & - & * & - \\
\hline Cassida panzeri Weise, 1907 & * & - & - & - & - \\
\hline Cassida rubiginosa O. F. Müller, 1776 & $*$ & - & - & - & - \\
\hline Cassida vibex Linnaeus, 1767 & * & $*$ & - & - & - \\
\hline Cassida viridis Linnaeus, 1758 & $*$ & $*$ & - & - & - \\
\hline Chaetocnema concinna (Marsham, 1802) & - & * & - & - & - \\
\hline Chrysolina fastuosa (Scopoli, 1763) & - & $* * *$ & - & - & - \\
\hline Chrysolina graminis (Linnaeus, 1758) & $* *$ & $*$ & - & - & - \\
\hline Chrysolina herbacea (Duftschmid, 1825) & $*$ & $* * *$ & - & - & - \\
\hline Chrysolina polita (Linnaeus, 1758) & - & $* *$ & - & - & - \\
\hline Chrysolina varians (Schaller, 1783) & - & $* *$ & - & $*$ & - \\
\hline Clytra quadripunctata (Linnaeus, 1758) & - & - & $* * *$ & - & - \\
\hline Coptocephala scopolina (Linnaeus, 1767) & $*$ & - & - & - & - \\
\hline Coptocephala unifasciata (Scopoli, 1763) & $*$ & - & - & - & - \\
\hline Crepidodera aurata (Marsham, 1802) & - & $* * *$ & - & - & - \\
\hline Cryptocephalus aureolus Suffrian, 1847 & - & - & $* *$ & - & - \\
\hline Cryptocephalus octopunctatus (Scopoli, 1763) & - & $* *$ & - & - & - \\
\hline Cryptocephalus quinquepunctatus (Scopoli, 1763) & - & $* *$ & - & $*$ & - \\
\hline Cryptocephalus virens Suffrian, 1847 & $*$ & - & - & - & - \\
\hline Derocrepis rufipes (Linnaeus, 1758) & - & $*$ & - & - & - \\
\hline Dibolia schillingii (Letzner, 1847) & $* * *$ & - & - & - & - \\
\hline Entomoscelis adonidis (Pallas, 1771) & $* * *$ & - & - & - & - \\
\hline Eumolpus asclepiadeus (Pallas, 1776) & $* *$ & - & - & - & - \\
\hline Exosoma collare (Hummel, 1825) & $*$ & - & - & - & - \\
\hline Galeruca dahlii (Joannis, 1865) & - & * & - & - & - \\
\hline Galeruca pomonae (Scopoli, 1763) & $* * *$ & - & - & - & - \\
\hline Galeruca tanaceti (Linnaeus, 1758) & $* *$ & - & - & - & - \\
\hline Galerucella lineola (Fabricius, 1781) & - & $*$ & - & - & - \\
\hline Gastrophysa viridula (De Geer, 1775) & * & ** & - & $*$ & $*$ \\
\hline Gonioctena interposita (Franz et Palmen, 1950) & $*$ & $*$ & - & - & - \\
\hline Hippuriphila modeeri (Linnaeus, 1761) & - & - & - & ** & - \\
\hline Labidostomis axillaris (Lacordaire, 1848) & $*$ & - & - & - & - \\
\hline Leptinotarsa decemlineata (Say, 1824) & $*$ & - & - & - & - \\
\hline Lilioceris merdigera (Linnaeus, 1758) & $* *$ & - & $*$ & - & - \\
\hline Lochmaea caprea (Linnaeus, 1758) & - & - & - & $* * *$ & - \\
\hline Lochmaea suturalis (C. G. Thomson, 1866) & - & $* *$ & - & $*$ & - \\
\hline Longitarsus echii (Koch, 1803) & $* * *$ & - & - & - & - \\
\hline Longitarsus luridus (Scopoli, 1763) & $* *$ & - & - & - & - \\
\hline Neocrepidodera femorata (Gyllenhal, 1813) & - & $* *$ & - & - & - \\
\hline Oreina cacaliae senecionis (Schummel, 1843) & - & - & $*$ & $* *$ & $* *$ \\
\hline Oreina intricata (Germar, 1824) & - & $*$ & - & ** & - \\
\hline Oreina virgulata praefica (Weise, 1884) & - & - & - & $* *$ & - \\
\hline Pachnephorus tessellatus (Duftschmid, 1825) & $*$ & - & - & - & - \\
\hline Pachybrachis fimbriolatus Suffrian, 1848 & ** & - & - & - & - \\
\hline Phaedon cochleariae (Fabricius, 1792) & - & - & - & $*$ & - \\
\hline Phratora vitellinae (Linnaeus, 1758) & $*$ & - & - & $*$ & - \\
\hline Phyllotreta nemorum (Linnaeus, 1758) & $* *$ & - & - & - & - \\
\hline Phyllotreta undulata Kutschera, 1860 & - & - & $* * *$ & - & - \\
\hline Plagiosterna aenea (Linnaeus, 1758) & - & $*$ & $*$ & - & - \\
\hline Plateumaris consimilis (Schrank, 1781) & - & - & $*$ & $*$ & $*$ \\
\hline Plateumaris rustica (Kunze, 1818) & - & $*$ & - & - & - \\
\hline Plateumaris sericea (Linnaeus, 1758) & - & $* * *$ & $* * *$ & - & - \\
\hline Plateumaris sp. & - & $*$ & - & - & - \\
\hline Prasocuris marginella (Linnaeus, 1758) & - & $*$ & - & - & - \\
\hline Sclerophaedon carpathicus (Weise, 1875) & - & - & - & - & $* *$ \\
\hline
\end{tabular}


Continuation of Table 2

\begin{tabular}{|c|c|c|c|c|c|}
\hline Species & Mezhyhirskyi Kamin & Zhenets & Dzhurdzhiy & Vesnarka & Pozhyzhevska \\
\hline Smaragdina salicina (Scopoli, 1763) & $*$ & - & - & - & - \\
\hline Timarcha goettingensis (Linnaeus, 1758) & $* * *$ & - & - & - & - \\
\hline Subtotal: & 30 & 25 & 8 & 13 & 4 \\
\hline \multicolumn{6}{|l|}{ CLERIDAE } \\
\hline Thanasimus formicarius (Linnaeus, 1758) & $*$ & - & - & $*$ & - \\
\hline Tillus elongatus (Linnaeus, 1758) & - & - & $*$ & - & - \\
\hline Trichodes apiarius (Linnaeus, 1758) & $* *$ & - & - & - & - \\
\hline Subtotal: & 2 & 0 & 1 & 1 & 0 \\
\hline \multicolumn{6}{|l|}{ COCCINELLIDAE } \\
\hline Adalia bipunctata (Linnaeus, 1758) & $* *$ & $* *$ & - & - & - \\
\hline Anatis ocellata (Linnaeus, 1758) & - & - & - & $*$ & - \\
\hline Calvia quatuordecimguttata (Linnaeus, 1758) & - & $*$ & $*$ & - & - \\
\hline Coccinella septempunctata Linnaeus, 1758 & $* * *$ & $* *$ & - & $* *$ & - \\
\hline Halyzia sedecimguttata (Linnaeus, 1758) & $*$ & - & - & - & - \\
\hline Harmonia axyridis (Pallas, 1773) & $* * *$ & $* * *$ & - & - & - \\
\hline Hippodamia variegata (Goeze, 1777) & $* *$ & - & - & $*$ & - \\
\hline Hyperaspis erytrocephala (Fabricius, 1787) & $* *$ & - & - & $*$ & - \\
\hline Nephus bipunctatus (Kugelann, 1794) & $*$ & - & - & - & - \\
\hline Nephus quadrimaculatus (Herbst, 1783) & $*$ & - & - & - & - \\
\hline Platynaspis luteorubra (Goeze, 1777) & $*$ & - & - & - & - \\
\hline Propylaea quatuordecimpunctata (Linnaeus, 1758) & ** & $*$ & $*$ & - & - \\
\hline Scymnus auritus Thunberg, 1795 & $*$ & - & - & - & - \\
\hline Scymnus frontalis (Fabricius, 1787) & - & - & - & $*$ & - \\
\hline Scymnus haemorrhoidalis Herbst, 1797 & $*$ & - & - & - & - \\
\hline Subtotal: & 12 & 5 & 2 & 5 & 0 \\
\hline \multicolumn{6}{|l|}{ CRYPTOPHAGIDAE } \\
\hline Cryptophagus subdepressus Gyllenhal, 1827 & - & $*$ & - & - & - \\
\hline Micrambe ulicis (Stephens, 1830) & $*$ & - & - & - & - \\
\hline Telmatophilus typhae (Fallén, 1802) & $*$ & - & - & - & - \\
\hline Subtotal: & 2 & 1 & 0 & 0 & 0 \\
\hline \multicolumn{6}{|l|}{ CURCULIONIDAE } \\
\hline Anoplus roboris Suffrian, 1840 & & $*$ & - & - & - \\
\hline Anthonomus rectirostris (Linnaeus, 1758) & $*$ & - & - & - & - \\
\hline Anthonomus rubi (Herbst, 1795) & $*$ & - & - & $*$ & - \\
\hline Archarius salicivorus (Paykull, 1792) & & $*$ & - & - & - \\
\hline Argoptochus quadrisignatus (Bach, 1856) & $*$ & - & - & - & - \\
\hline Bryodaemon hanakii (J. Frivaldszky, 1865) & - & $*$ & - & - & - \\
\hline Centricnemus leucogrammus (Germar, 1824) & $* *$ & - & - & - & - \\
\hline Ceutorhynchus obstrictus (Marsham, 1802) & $* *$ & - & - & - & - \\
\hline Cionus hortulanus (Geoffroy, 1785) & - & $*$ & - & - & - \\
\hline Cionus tuberculosus (Scopoli, 1763) & - & $*$ & - & - & - \\
\hline Cleonis pigra (Scopoli, 1763) & $* *$ & - & - & - & - \\
\hline Cleopomiarus graminis (Gyllenhal, 1813) & $*$ & - & - & - & - \\
\hline Cyphocleonus dealbatus (Gmelin, 1790) & $* * *$ & - & - & - & - \\
\hline Donus comatus (Boheman, 1842) & - & $* *$ & - & $*$ & - \\
\hline Donus ovalis (Boheman, 1842) & - & $* *$ & - & - & - \\
\hline Donus rubi (Krauss, 1900) & - & $* * *$ & - & $*$ & - \\
\hline Donus velutinus (Boheman, 1842) & - & $* *$ & - & - & - \\
\hline Eusomus ovulum Germar, 1824 & $*$ & - & - & - & - \\
\hline Foucartia squamulata (Herbst, 1795) & $* *$ & $*$ & - & - & - \\
\hline Gymnetron melanarium (Germar, 1821) & $*$ & - & - & - & - \\
\hline Hylastes cunicularius Erichson, 1836 & - & - & $*$ & - & - \\
\hline Hylobius abietis (Linnaeus, 1758) & $*$ & $* * *$ & - & - & - \\
\hline Hypera rumicis (Linnaeus, 1758) & $*$ & - & - & - & - \\
\hline Hypera transsylvanica (Petri, 1901) & $* *$ & - & - & - & - \\
\hline Isochnus foliorum (O. F. Müller, 1764) & - & $*$ & - & - & - \\
\hline Larinus obtusus Gyllenhal, 1835 & $* * *$ & - & - & $*$ & - \\
\hline Larinus sturnus (Schaller, 1783) & $* *$ & - & - & - & - \\
\hline Larinus turbinatus Gyllenhal, 1835 & $* *$ & - & - & - & - \\
\hline Liophloeus gibbus Boheman,1842 & $*$ & - & - & - & - \\
\hline Liophloeus liptoviensis Weise, 1894 & $*$ & $*$ & - & $*$ & $*$ \\
\hline Liophloeus tessulatus (O. F. Müller, 1776) & $* *$ & - & - & $*$ & - \\
\hline Liparus coronatus (Goeze, 1777) & $*$ & - & - & - & - \\
\hline
\end{tabular}


A. M. SUMAROKOV, A. M. ZAMOROKA Spatial patterns and habitat differentiation of the beetle (Insecta: Coleoptera) fauna of protected areas in the Eastern Carpathians (Ivano-Frankivsk Region, Ukraine)

Continuation of Table 2

\begin{tabular}{|c|c|c|c|c|c|}
\hline $\begin{array}{r}\text { Species } \\
\end{array}$ & Mezhyhirskyi Kamin & Zhenets & Dzhurdzhiy & Vesnarka & Pozhyzhevska \\
\hline Liparus glabrirostris (Küster, 1849) & - & $* * *$ & ** & - & * \\
\hline Lixus bardanae (Fabricius, 1787) & * & - & - & - & - \\
\hline Lixus iridis Olivier, 1807 & $* * *$ & - & - & - & - \\
\hline Lixus punctiventris Boheman, 1835 & ** & - & - & - & - \\
\hline Magdalis armigera (Geoffroy, 1785) & $*$ & - & - & - & - \\
\hline Magdalis ruficornis (Linnaeus, 1758) & - & - & * & - & - \\
\hline Mecaspis alternans (Hellwig, 1795) & $* * *$ & - & - & - & - \\
\hline Miarus ajugae (Herbst, 1795) & $*$ & - & - & - & - \\
\hline Miarus simplex Solari, 1947 & - & * & - & - & - \\
\hline Microplontus campestris (Gyllenhal, 1837) & * & - & - & - & - \\
\hline Nedyus quadrimaculatus (Linnaeus, 1758) & * & - & * & * & - \\
\hline Otiorhynchus asplenii Miller, 1868 & - & - & - & - & * \\
\hline Otiorhynchus coecus coecus Germar, 1824 & - & - & ** & ** & * \\
\hline Otiorhynchus deubeli Ganglbauer, 1896 & - & *** & - & $*$ & - \\
\hline Otiorhynchus fullo (Schrank, 1781) & ** & - & - & - & - \\
\hline Otiorhynchus krattereri Boheman, 1843 & - & * & - & - & * \\
\hline Otiorhynchus kuenburgi Stierlin, 1866 & - & * & - & - & - \\
\hline Otiorhynchus morio (Fabricius, 1781) & - & - & - & - & * \\
\hline Otiorhynchus multipunctatus (Fabricius, 1792) & - & - & **** & - & - \\
\hline Otiorhynchus nodosus (O. F. Müller, 1764) & - & - & ** & ** & - \\
\hline Otiorhynchus obsidianus Boheman, 1843 & - & * & - & - & - \\
\hline Otiorhynchus opulentus Germar, 1836 & - & $*$ & - & - & - \\
\hline Otiorhynchus ovatus (Linnaeus 1758) & ** & - & **** & - & - \\
\hline Otiorhynchus peneckianus Smreczyński, 1963 & - & *** & - & - & - \\
\hline Otiorhynchus raucus (Fabricius, 1777) & $* * *$ & - & - & - & - \\
\hline Otiorhynchus tenebricosus (Herbst, 1784) & - & $* * *$ & $* *$ & * & * \\
\hline Otiorhynchus singularis (Linnaeus, 1767) & - & - & - & - & ** \\
\hline \begin{tabular}{|l|} 
Otiorhynchus velutinus Germar, 1824 \\
\end{tabular} & $* * *$ & - & - & - & - \\
\hline Paophilus afflatus (Boheman, 1833) & $*$ & - & - & - & - \\
\hline Phyllobius argentatus (Linnaeus, 1758) & **** & - & - & * & - \\
\hline Phyllobius brevis Gyllenhal, 1834 & $* *$ & - & - & - & - \\
\hline Phyllobius contemptus Schönherr, 1832 & ** & - & - & - & - \\
\hline Phyllobius glaucus (Scopoli, 1763) & * & * & - & * & - \\
\hline Phyllobius maculicornis Germar, 1824 & - & - & - & *** & - \\
\hline Phyllobius oblongus (Linnaeus, 1758) & ** & - & $*$ & - & - \\
\hline Phyllobius pomaceus Gyllenhal, 1834 & ** & - & - & - & - \\
\hline Phyllobius pyri (Linnaeus, 1758) & $* * *$ & - & - & - & - \\
\hline Plinthus sturmii Germar, 1819 & - & - & - & * & - \\
\hline Plinthus tischeri Germar, 1824 & - & - & * & * & - \\
\hline Polydrusus amoenus (Germar, 1824) & - & - & - & $*$ & - \\
\hline Polydrusus fulvicornis (Fabricius, 1792) & - & * & - & - & - \\
\hline Polydrusus inustus Germar, 1824 & - & - & $\overline{-}$ & * & - \\
\hline Polydrusus mollis (Ström, 1768) & - & - & - & * & - \\
\hline Polydrusus picus (Fabricius, 1792) & * & - & - & - & - \\
\hline Polydrusus pilosus Gredler, 1866 & * & * & - & * & - \\
\hline Polydrusus pterygomalis Boheman, 1840 & * & - & - & - & - \\
\hline Rhinoncus bruchoides (Herbst, 1784) & * & - & - & - & - \\
\hline Rhinoncus leucostigma (Marsham, 1802) & * & * & - & * & - \\
\hline Rhinoncus pericarpius (Linnaeus, 1758) & * & - & $\overline{-}$ & $\overline{-}$ & - \\
\hline Sciaphilus asperatus (Bonsdorff, 1785) & * & - & - & - & - \\
\hline Scleropterus serratus (Germar, 1824) & - & $*$ & - & - & - \\
\hline Sibinia pellucens (Scopoli 1772) & * & - & - & - & - \\
\hline Sitona ambiguus Gyllenhal, 1834 & * & - & - & - & - \\
\hline Sitona inops Gyllenhal, 1832 & $* *$ & - & - & - & - \\
\hline Sitona languidus Gyllenhal, 1834 & **** & - & - & - & - \\
\hline Sitona lineatus (Linnaeus, 1758) & - & ** & - & - & - \\
\hline Sitona longulus Gyllenhal, 1834 & $*$ & - & $\begin{array}{l}- \\
\end{array}$ & $\begin{array}{l}- \\
\end{array}$ & - \\
\hline Sitona striatellus Gyllenhal, 1834 & $* * *$ & - & - & - & - \\
\hline Sitona sulcifrons (Thunberg, 1798) & * & - & - & - & - \\
\hline Sitona suturalis Stephens, 1831 & $*$ & - & - & - & - \\
\hline Sitona waterhousei Walton, 1846 & $* * *$ & - & - & - & - \\
\hline Smicronyx jungermanniae (Reich, 1797) & $*$ & * & - & - & - \\
\hline Smicronyx reichii (Gyllenhal, 1836) & * & - & - & - & - \\
\hline Stephanocleonus microgrammus (Gyllenhal, 1834) & ** & - & - & - & - \\
\hline
\end{tabular}


Continuation of Table 2

Species

Strophosoma melanogrammus (Förster, 1771)

Tachyerges pseudostigma (Tempere, 1982)

Tachyerges salicis (Linnaeus, 1758)

Tanymecus palliatus (Fabricius, 1787)

Tapinotus sellatus (Fabricius, 1794)

Trichosirocalus troglodytes (Fabricius, 1787)

Tychius breviusculus Desbrochers, 1873

Tychius medicaginis C. N. F. Brisout de Barneville, 1862

Tychius meliloti Stephens, 1831

Tychius picirostris (Fabricius, 1787)

Tychius stephensi Schönherr, 1836

Anthrenus pimpinellae (Fabricius, 1775)

Anthrenus scrophulariae (Linnaeus, 1758)

Attagenus pellio (Linnaeus, 1758)

Dermestes laniarius Illiger, 1802

\section{DRYOPIDAE \\ Dryops ernesti Des Gozis, 1886 \\ Dryops striatopunctatus (Heer, 1841)

\begin{tabular}{|l|}
\hline \multicolumn{1}{|c}{ DYTISCIDAE } \\
\hline Agabus bipustulatus (Linnaeus, 1767) \\
\hline Ilybius fuliginosus (Fabricius, 1792) \\
\hline Ilybius quadriguttatus (Lacordaire, 1835) \\
\hline Platambus maculatus (Linnaeus, 1758) \\
\hline
\end{tabular} \\ Platambus maculatus (Linnaeus, 1758)}

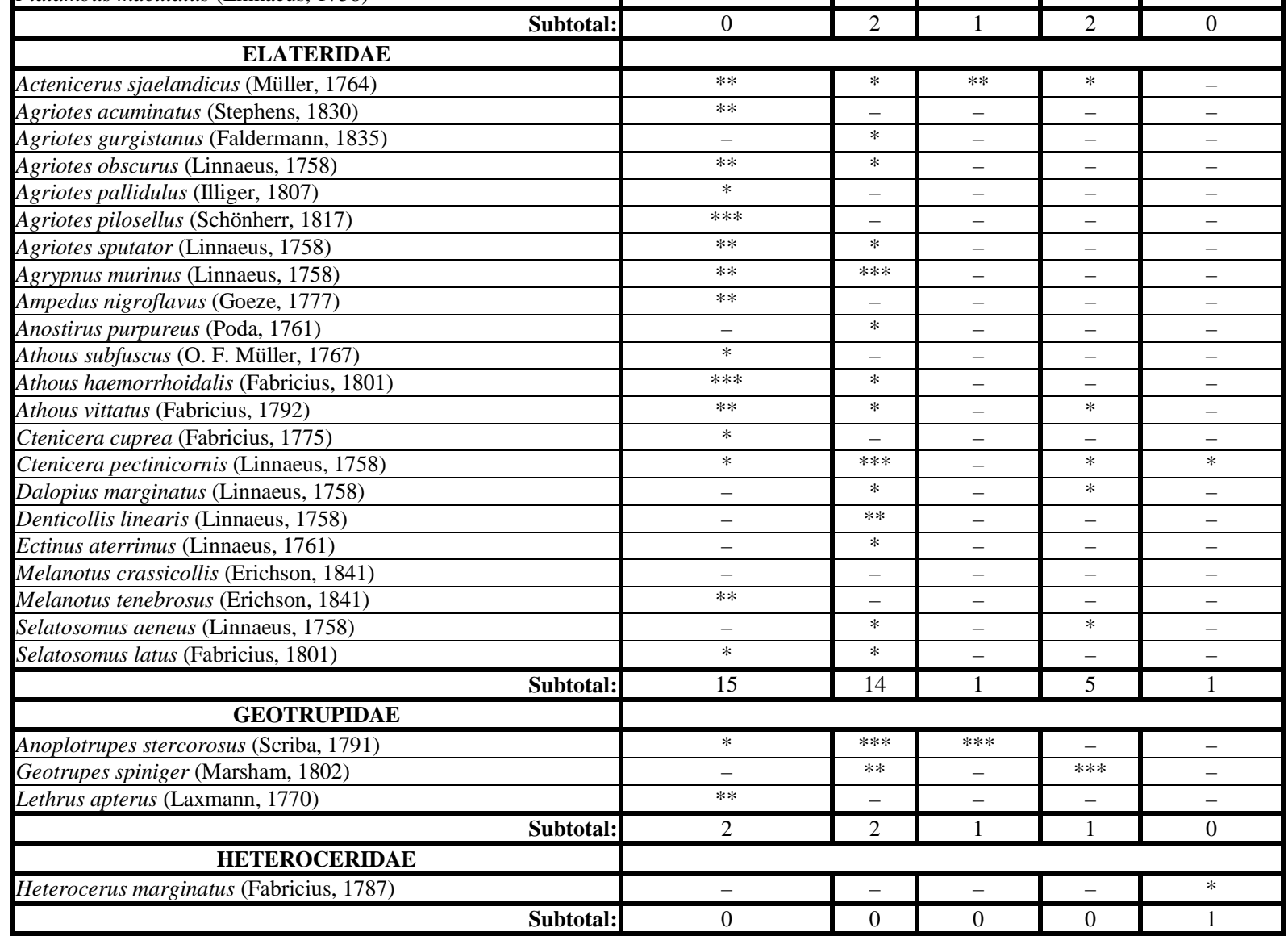


A. M. SUMAROKOV, A. M. ZAMOROKA

Spatial patterns and habitat differentiation of the beetle (Insecta: Coleoptera) fauna of protected areas in the Eastern Carpathians (Ivano-Frankivsk Region, Ukraine)

Continuation of Table 2

\begin{tabular}{|c|c|c|c|c|c|}
\hline Species & Mezhyhirskyi Kamin & Zhenets & Dzhurdzhiy & Vesnarka & Pozhyzhevska \\
\hline \multicolumn{6}{|l|}{ HYDROPHILIDAE } \\
\hline Cercyon analis (Paykull, 1798) & $*$ & - & - & - & - \\
\hline Hydrobius fuscipes (Linnaeus, 1758) & $*$ & - & - & - & - \\
\hline Sphaeridium scarabaeoides (Linnaeus, 1758) & - & - & ** & - & - \\
\hline Subtotal: & 2 & 0 & 1 & 0 & 0 \\
\hline \multicolumn{6}{|l|}{ LATRIDIIDAE } \\
\hline Corticaria impressa (Olivier, 1790) & $* *$ & - & - & - & - \\
\hline Corticarina similata (Gyllenhal, 1827) & $*$ & - & - & - & - \\
\hline Cortinicara gibbosa (Herbst, 1793) & - & - & - & $*$ & - \\
\hline Subtotal: & 2 & 0 & 0 & 1 & 0 \\
\hline \multicolumn{6}{|l|}{ LYCIDAE } \\
\hline Dictyoptera aurora (Herbst, 1784) & - & - & $*$ & - & - \\
\hline Lopheros rubens (Gyllenhal, 1817) & - & $*$ & $*$ & - & - \\
\hline Subtotal: & 0 & 1 & 2 & 0 & 0 \\
\hline \multicolumn{6}{|l|}{ LYMEXILIDAE } \\
\hline Hylecoetus dermestoides (Linnaeus, 1761) & $*$ & - & - & - & - \\
\hline Subtotal: & 1 & 0 & 0 & 0 & 0 \\
\hline \multicolumn{6}{|l|}{ MELANDRYIDAE } \\
\hline Melandrya dubia (Schaller, 1783) & - & $*$ & - & - & - \\
\hline Subtotal: & 0 & 1 & 0 & 0 & 0 \\
\hline \multicolumn{6}{|l|}{ MELYRIDAE } \\
\hline Charopus concolor (Fabricius, 1801) & $*$ & - & - & - & - \\
\hline Charopus flavipes (Paykull, 1798) & * & - & - & - & - \\
\hline Charopus pallipes (Olivier, 1790) & $*$ & - & - & - & - \\
\hline Danacea nigritarsis (Küster, 1850) & * & - & - & - & - \\
\hline Dasytes caeruleus (De Geer, 1774) & - & $*$ & - & - & - \\
\hline Dasytes fusculus (Illiger, 1801) & - & $*$ & - & - & - \\
\hline Dasytes plumbeus (O. F. Müller, 1776) & $*$ & - & $*$ & - & - \\
\hline Dolichosoma lineare (Rossi, 1794) & $* * *$ & $* *$ & $* *$ & - & - \\
\hline Malachius aeneus (Linnaeus, 1758) & - & $* *$ & - & $*$ & - \\
\hline Malachius bipustulatus (Linnaeus, 1758) & $* * *$ & $*$ & $* *$ & - & - \\
\hline Subtotal: & 7 & 5 & 3 & 1 & 0 \\
\hline \multicolumn{6}{|l|}{ NITIDULIDAE } \\
\hline Cychramus variegatus (Herbst, 1792) & - & $*$ & $*$ & - & - \\
\hline Epuraea marseuli Reitter, 1872 & - & - & - & - & $*$ \\
\hline Glischrochilus quadrisignatus (Say, 1835) & $* *$ & - & - & - & - \\
\hline Meligethes atratus (Olivier, 1790) & - & - & - & - & $*$ \\
\hline Omosita colon (Linnaeus, 1758) & $*$ & - & - & - & - \\
\hline Subtotal: & 2 & 1 & 1 & 0 & 2 \\
\hline \multicolumn{6}{|l|}{ OEDEMERIDAE } \\
\hline Anogcodes ustulatus (Scopoli, 1763) & * & * & - & - & - \\
\hline Chrysanthia viridissima (Linnaeus, 1758) & - & - & - & - & $*$ \\
\hline Oedemera femorata (Scopoli, 1763) & $* * *$ & $*$ & - & - & - \\
\hline Oedemera lurida (Marsham, 1802) & - & $*$ & - & - & - \\
\hline Oedemera virescens (Linnaeus, 1767) & - & $* *$ & - & - & - \\
\hline Subtotal: & 2 & 4 & 0 & 0 & 1 \\
\hline \multicolumn{6}{|l|}{ ORSODACNIDAE } \\
\hline Orsodacne cerasi (Linnaeus, 1758) & - & $*$ & - & - & - \\
\hline Subtotal: & 0 & 1 & 0 & 0 & 0 \\
\hline \multicolumn{6}{|l|}{ PTINIDAE } \\
\hline Lasioderma redtenbacheri (Bach, 1852) & - & - & - & - & $*$ \\
\hline Lasioderma serricorne (Fabricius, 1792) & - & $*$ & - & - & - \\
\hline Subtotal: & 0 & 1 & 0 & 0 & 1 \\
\hline \multicolumn{6}{|l|}{ PYROCHROIDAE } \\
\hline Schizotus pectinicornis (Linnaeus, 1758) & - & - & $*$ & - & - \\
\hline Subtotal: & 0 & 0 & 1 & 0 & 0 \\
\hline \multicolumn{6}{|l|}{ SCARABAEIDAE } \\
\hline Amphimallon solstitiale (Linnaeus, 1758) & $* * *$ & - & - & - & - \\
\hline Aphodius abdominalis (Bonelli, 1812) & - & - & - & - & $*$ \\
\hline Cetonia aurata aurata (Linnaeus, 1758) & $* *$ & $* *$ & - & - & - \\
\hline Copris lunaris (Linnaeus, 1758) & $*$ & - & - & - & - \\
\hline
\end{tabular}


Continuation of Table 2

\begin{tabular}{|c|c|c|c|c|c|}
\hline $\begin{array}{c}\text { Species } \\
\end{array}$ & Mezhyhirskyi Kamin & Zhenets & Dzhurdzhiy & Vesnarka & Pozhyzhevska \\
\hline Melolontha melolontha (Linnaeus, 1758) & $* * *$ & - & - & - & - \\
\hline Oxythyrea funesta (Poda, 1761) & $* *$ & $* *$ & - & - & - \\
\hline Phyllopertha horticola (Linnaeus, 1758) & $* * *$ & $*$ & - & - & - \\
\hline Trichius fasciatus (Linnaeus, 1758) & - & $*$ & $*$ & - & - \\
\hline Tropinota hirta (Poda, 1761) & $* * *$ & - & - & - & - \\
\hline Valgus hemipterus (Linnaeus, 1758) & $* *$ & - & - & - & - \\
\hline Subtotal: & 8 & 4 & 1 & 0 & 1 \\
\hline \multicolumn{6}{|l|}{ SCRAPTIIDAE } \\
\hline Anaspis frontalis (Linnaeus, 1758) & - & - & $*$ & $*$ & $*$ \\
\hline Subtotal: & 0 & 0 & 1 & 1 & 1 \\
\hline \multicolumn{6}{|l|}{ SILPHIDAE } \\
\hline Nicrophorus germanicus (Linnaeus, 1758) & $*$ & - & - & - & - \\
\hline Nicrophorus humator (Gleditsch, 1767) & $*$ & - & - & - & - \\
\hline Nicrophorus interruptus (Stephens, 1830) & $*$ & - & - & - & - \\
\hline Nicrophorus vespillo (Linnaeus, 1758) & $* * *$ & - & - & - & - \\
\hline Nicrophorus vespilloides Herbst, 1783 & $* * *$ & - & - & - & - \\
\hline Oiceoptoma thoracicum (Linnaeus, 1758) & $*$ & - & $* *$ & - & - \\
\hline Phosphuga atrata (Linnaeus, 1758) & $*$ & $*$ & - & - & - \\
\hline Silpha carinata Herbst, 1783 & $* * *$ & - & $* *$ & - & - \\
\hline Silpha perforata Gebler, 1832 & - & $*$ & - & - & - \\
\hline Thanatophilus rugosus (Linnaeus, 1758) & $*$ & - & - & - & - \\
\hline Thanatophilus sinuatus (Fabricius, 1775) & $* *$ & - & - & - & - \\
\hline Subtotal: & 10 & 2 & 2 & 0 & 0 \\
\hline \multicolumn{6}{|l|}{ SILVANIDAE } \\
\hline Silvanoprus fagi (Guérin-Ménéville, 1844) & - & - & - & $*$ & - \\
\hline Subtotal: & 0 & 0 & 0 & 1 & 0 \\
\hline \multicolumn{6}{|l|}{ STAPHYLINIDAE } \\
\hline Amphichroum canaliculatum (Erichson, 1840) & $* *$ & - & - & $*$ & - \\
\hline Anotylus rugosus (Fabricius, 1775) & $*$ & - & - & - & - \\
\hline Deleaster dichrous (Gravenhorst, 1802) & $* *$ & $* *$ & $*$ & - & - \\
\hline Drusilla canaliculata (Fabricius, 1787) & $* * *$ & $*$ & - & - & - \\
\hline Eusphalerum longipenne (Erichson, 1839) & $*$ & - & - & - & - \\
\hline Eusphalerum sp. & $*$ & - & - & - & - \\
\hline Geodromicus sp. & $*$ & - & - & - & - \\
\hline Gyrohypnus sp. & $*$ & - & - & - & - \\
\hline Ochthephilus sp. & $* *$ & - & - & - & - \\
\hline Ocypus macrocephalus (Gravenhorst, 1802) & - & $*$ & $*$ & - & - \\
\hline Ocypus ormayi (Reitter, 1887) & $*$ & $*$ & - & $* *$ & - \\
\hline Ocypus tenebricosus (Gravenhorst, 1846) & * & * & $*$ & - & - \\
\hline Ontholestes haroldi (Eppelsheim, 1884) & $*$ & - & - & - & - \\
\hline Ontholestes murinus (Linnaeus, 1758) & - & $*$ & - & - & - \\
\hline Paederus fuscipes Curtis, 1826 & - & $*$ & - & - & - \\
\hline Paederus littoralis Gravenhorst, 1802 & $*$ & $*$ & - & - & - \\
\hline Paederidus ruficollis (Fabricius, 1777) & - & $*$ & - & - & - \\
\hline Pella humeralis (Gravenhorst, 1802) & $*$ & - & - & - & - \\
\hline Pella limbata (Paykull, 1789) & $* *$ & - & - & - & - \\
\hline Philonthus addendus Sharp, 1867 & - & $*$ & - & - & - \\
\hline Philonthus decorus (Gravenhorst, 1802) & $* *$ & $* *$ & $* * *$ & $* *$ & - \\
\hline Platydracus fulvipes (Scopoli, 1763) & - & $*$ & - & - & - \\
\hline Platydracus latebricola (Gravenhorst, 1806) & $*$ & $*$ & - & - & - \\
\hline Platydracus stercorarius (Olivier, 1795) & $*$ & * & - & - & - \\
\hline Quedius transsylvanicus Weise, 1875 & - & - & - & $*$ & - \\
\hline Staphylinus caesareus Cederhjelm, 1798 & $*$ & - & - & - & - \\
\hline Stenus similis (Herbst, 1784) & $*$ & - & - & $*$ & - \\
\hline Tachinus rufipes (Linnaeus, 1758) & $*$ & $*$ & - & $*$ & - \\
\hline Tasgius melanarius (Heer, 1839) & $*$ & - & - & - & - \\
\hline Xantholinus dvoraki Coiffait, 1956 & ** & - & - & - & - \\
\hline Subtotal: & 23 & 15 & 4 & 6 & 0 \\
\hline \multicolumn{6}{|l|}{ TENEBRIONIDAE } \\
\hline Allecula morio (Fabricius, 1787) & $*$ & - & - & - & - \\
\hline Bolitophagus reticulatus (Linnaeus, 1767) & - & - & $*$ & - & - \\
\hline Blaps lethifera Marsham, 1802 & $*$ & - & - & - & - \\
\hline Cteniopus sulphureus (Linnaeus, 1758) & - & $*$ & - & $*$ & - \\
\hline
\end{tabular}


A. M. SUMAROKOV, A. M. ZAMOROKA Spatial patterns and habitat differentiation of the beetle (Insecta: Coleoptera) fauna of protected areas in the Eastern Carpathians (Ivano-Frankivsk Region, Ukraine)

Continuation of Table 2

\begin{tabular}{|c|c|c|c|c|c|}
\hline Species & Mezhyhirskyi Kamin & Zhenets & Dzhurdzhiy & Vesnarka & Pozhyzhevska \\
\hline Lagria hirta (Linnaeus, 1758) & $* *$ & - & $*$ & $*$ & - \\
\hline Opatrum sabulosum (Linnaeus, 1761) & $* * *$ & - & - & - & - \\
\hline Pedinus femoralis (Linnaeus, 1767) & $* * *$ & - & - & - & - \\
\hline Stenomax aeneus (Scopoli, 1763) & * & - & - & - & - \\
\hline Subtotal: & 6 & 1 & 2 & 2 & 0 \\
\hline \multicolumn{6}{|l|}{ THROSCIDAE } \\
\hline Trixagus dermestoides (Linnaeus, 1766) & - & - & - & $*$ & - \\
\hline Subtotal: & 0 & 0 & 0 & 1 & 0 \\
\hline Total: & 327 & 280 & 192 & 92 & 48 \\
\hline
\end{tabular}

No t e s : * — solitary records; ** — common; *** — abundant.

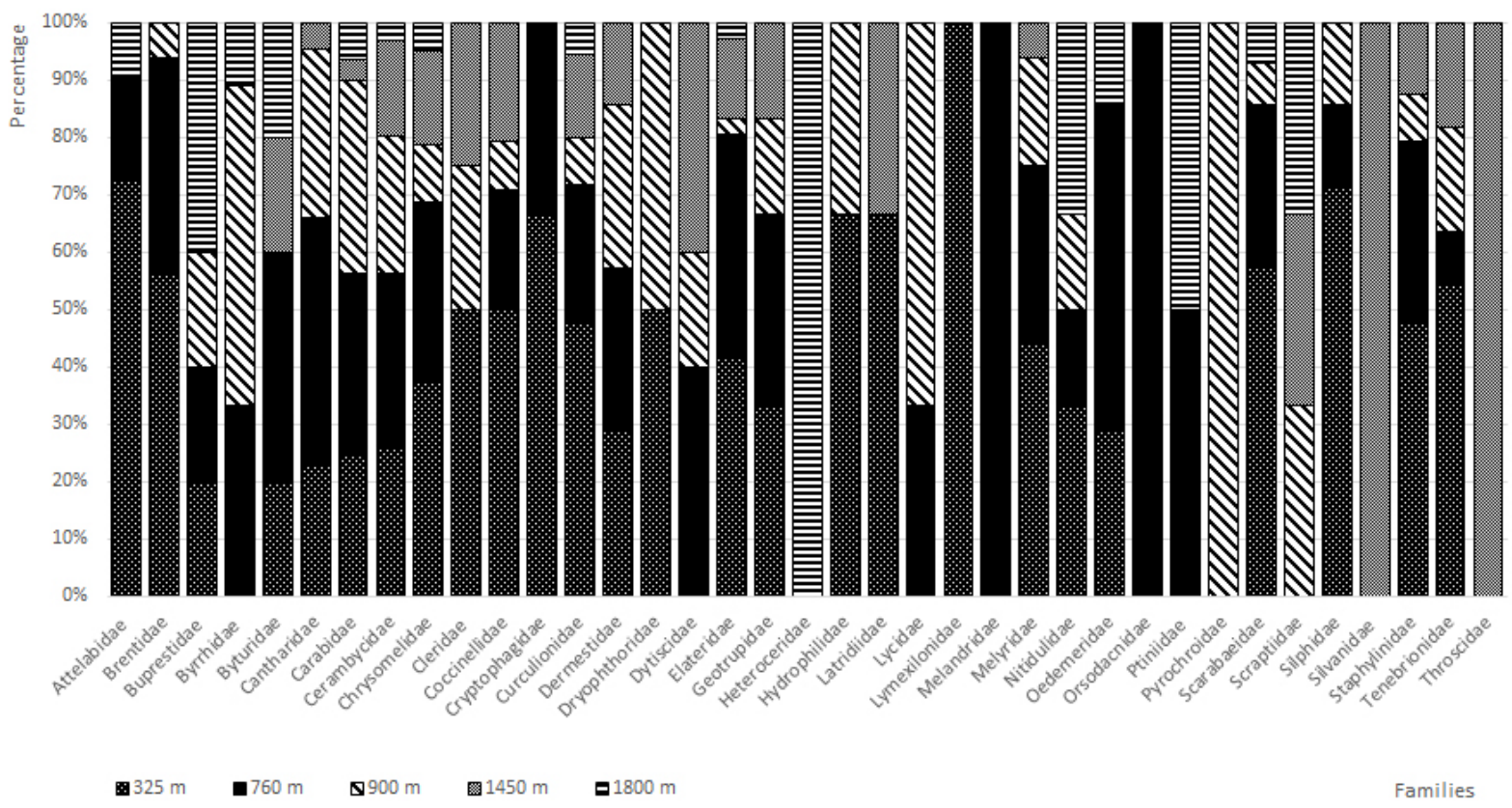

F i g. 1. Percentage variation of taxonomic (families) composition of the studied Coleoptera fauna.

Trophic c omposition of studied Coleoptera fauna represents all types of feeding specialization. Phytophagous species (including herbivorous, palynophagous, xylophagous, bryophagous, etc.) constitute $45.5 \%$ of Coleoptera fauna of the region. The phytophages represent mainly by Curculionidae, Chrysomelidae, Cerambycidae, Elateridae, and Tenebrionidae. Carnivorous Coleoptera constitutes 38.3\% of the studied species and represent mainly by Carabidae, Cantaridae, Coccinellidae, and Staphylinidae. Finally, species with other feeding specialization (e.g., coprophagous, necrophagous, detritophagous, mycetophagous, myxophagous, commensals) constitute $16.2 \%$ of Coleoptera fauna and represent mainly by Scarabaeidae, Silphidae, Dermestidae, Stapylinidae, Cryptophagidae, and Melandryidae.

Spatial distribution. The composition of Coleoptera fauna, species richness, and general biodiversity vary due to the regional altitudinal ecological gradient. The general trend of the regional altitudinal ecological gradient extends on over $100 \mathrm{~km}$ and directed from the south-west (mountains) to north-east (lowlands) within the studied territory. It causes synchronous changes of the main ecological variables, including altitude (decreases), annual temperature (increases), annual precipitation (decreases), annual air and soil humidity (decrease), annual duration, and intensity of solar insolation (increase). These lead to the formation of $\beta$-diversity of beetles and their spatial distribution within different types of ecosystems.

The regional altitudinal ecological gradient strongly impacts on $\beta$-diversity and species richness of beetles within the studied territory (Fig. 2). In general, species richness gradually decreases along with increasing altitude. The highest species richness (327 species) is in lowlands at $325 \mathrm{~m} \mathrm{a}$. s. l. At an altitude of $760 \mathrm{~m}$ a. s. 1 ., we found 280 species, and significantly fewer species (192) were identified at an altitude of $900 \mathrm{~m}$ a. s. 1 . Species richness decreases sharply when altitude exceeds 1,000 m a. s. l. For instance, we collected 92 species at an 
altitude of 1,450 $\mathrm{m}$ a. s. l. and 48 species at $1,800 \mathrm{~m}$ a. s. 1 . Our data suggest that species richness is changing at least seven times under the impact of the regional altitudinal ecological gradient.

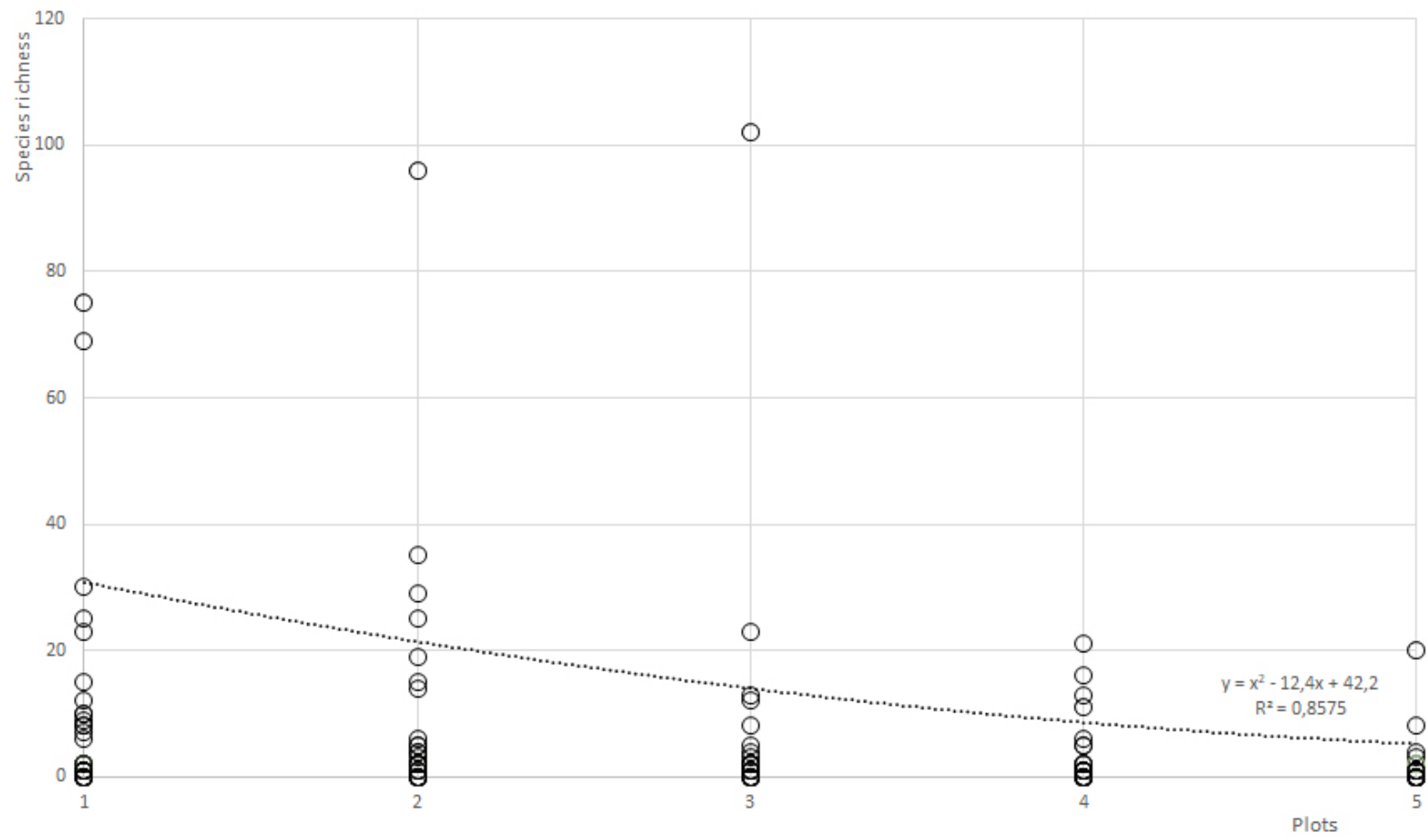

Fig. 2. Changing of Coleoptera species richness within sample plots: 1 - Mezhyhirskyi Kamin, 325 m a. s. l.; 2 - Zhenets, 760 m a. s. l.; 3 - Dzhudzhiy, $900 \mathrm{~m}$ a. s. l.; 4 - Vesnarka, 1450 m a. s. l.; 5 - Pozhyzhevska, 1800 m a. s. l.

Altitudinal ecological gradient also affects species composition. Ubiquist species consist of only $3.5 \%$ of all registered beetles. These include Abax parallelopipedus, Bembidion geniculatum, Carabus violaceus, Carabus glabratus, Carabus caraboides, Notiophilus palustris, Anastrangalia sanquinolenta, Leptura annularis, Rhagium inquisitor, Stictoleptura rubra, Tetropium castaneum, Gastrophysa viridula, Cocinella septempunctata, Harmonia axyridis, Liophloeus liptoviensis, Actenicerus sjaelandicus, Ctenicera pectinicornis, Lagria hirta, Anoplotrupes stercorosus, Philonthus decorus.

We found 327 species of beetles for the steppe ecosystems, which consist of nemoral species (81\%), steppic species (7\%) and boreo-montane species (5\%) on the easternmost foothills of the Eastern Carpathians. The remain $7 \%$ of species are ubiquists. The specific steppic speciesare represernted by Carabus excellens, Ophonus rupicola, Ophonus rufibarbis, Calamobius filum, Dorcadion fulvum, Dorcadion holosericeum, Phytoecia coerulescens, Phytoecia tigrina, Phytoecia uncinata, Dibolia schillingii, Entomoscelis adonidis, Eumolpus asclepiadeus, Liparus coronatus, Stephanocleonus microgrammus, Lethrus apterus, Copris lunaris, Tropinota hirta, Thanatophilus sinuatus, Thanatophilus rugosus, Blaps lethifera, Opatrum sabulosum, Pedinus femoralis, Xantholinus dvoraki.

In the mountain part of the region we identified 435 species including boreo-montane species (63\%), nemoral species (31\%), alpine species (1.3\%), and ubiquists (4.7\%). Alpine species are the most specific for the region. These include Otiorhynchus asplenii, Ancistronycha abdominalis, Carabus rothi, Carabus sylvestris, Carabus fabricii, Nebria transsylvanica.

Habitat differentiation. Our findings showed that Coleoptera fauna is highly differentiated with a low similarity within habitats (Fig. 3). In particular, Coleoptera fauna of the steppes is only $20 \%$ similar to the fauna of fir-beech forests (share 101 species) and much low in comparison with the fauna in other studied habitats. The least similar (2.2\%) faunas of steppes and alpine meadows, sharing only 8 species (all are ubiquitous). Surprisingly, however, Coleoptera faunas of secondary fir-beech forests and primeval beech-fir forests are similar only on $29 \%$ (share 106 species). We believe that a possible explanation is the ongoing recovery processes in secondary forests in response to their past exploitation before conservation. The fauna of high mountains pastures is very different from other habitats. It is $15 \%$ similar to forest habitats, and $13 \%$ - to alpine meadows. 


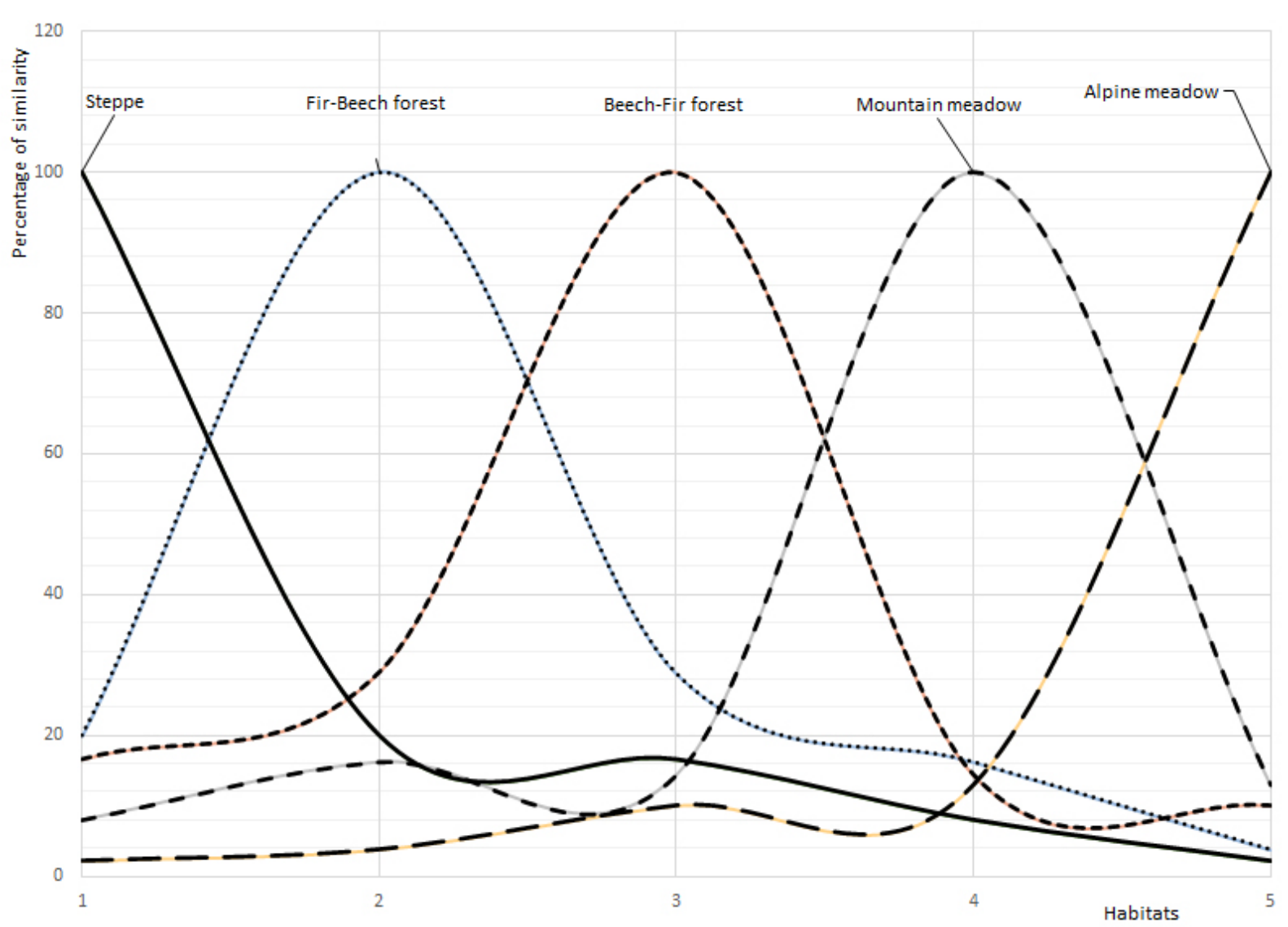

F i g. 3. Habitat differentiation of Coleoptera fauna within sample plots: 1 - Mezhyhirskyi Kamin, 325 m a. s. l.; 2 - Zhenets, 760 m a. s. l.; 3 - Dzhudzhiy, $900 \mathrm{~m}$ a. s. l.; 4 - Vesnarka, 1450 m a. s. l.; 5 - Pozhyzhevska, 1800 m a. s. l.

The most common and abundant species on steppes are C. obscura, C. rufa, Rh. fulva, A. aenea, C. fossor, D. dentate, H. calceatus, L. ferrugineus, O. rupicola, T. quadristriatus, A. cardui, C. filum, D. fulvum, D. holosericeum, O. erythrocephala, Ph. coerulescens, S. attenuata, A. quadrimaculata, D. schillingii, E. adonidis, E. asclepiadeus, G. pomonae, L.echii, T. goettingensis, C. campestris, C. septempunctata, H. axyridis, C. dealbatus, L. obtusus, L. iridis, M. alternans, O. velutinus, O. raucus, O. ovatus, Ph. argentatus, Ph. pyri, S. languidus, S. striatellus, S. waterhousei, T. palliates, D. laniarius, A. pilosellus, A. murinus, A. haemorrhoidalis, D. lineare, M. bipustulatus, A. solstitiale, M. melolontha, P. horticola, T. hirta, N. vespillo, $N$. vespilloides, S. carinata, Th. sinuatus, D. canaliculata, X. dvoraki, O. sabulosum, P. femoralis.

Typical species for the secondary fir-beech forest are C. annularis, C. nigricans, A. parallelopipedus, B. quadrimaculatum, B. lunatum, C. auronitens, C. violaceus, C. obsoletus, C. glabratus, C. linnei, C. coriaceus, D. carpathicus, P. assimilis, P. foveolatus, A.tabacicolor, C. virginea, D. collaris, E. clathratus, L. quadrifasciata, L. virens, M. minor, M. sartor, M. sutor, O. brunneum, P. cerambyciformis, P. lurida, Rh. inquisitor, S. melanura, S. rubra, T. castaneum, A. alni, C. fastuosa, C. herbacea, C. aurata, N. femorata, P. sericea, C. septempunctata, H. axyridis, D. rubi, H. abietis, L. glabrirostris, O. tenebricosus, O. ovatus, S. lineatus, A.polonicus, A. scrophulariae, A.murinus, C.pectinicornis, D. lineare, M. bipustulatus, G. quadrisignatus, O. femorata, G. stercorosus, G. spiniger, Ph. decorus.

In the primeval beech-fir forests the most abundant species are C. annularis, A. parallelopipedus, C. metallicus, C. auronitens, C. violaceus, C. obsoletus, C. glabratus, C. linnei, C. coriaceus, P. assimilis, P. pilosus, A. tabacicolor, C. virginea, D. collaris, E. clathratus, L. quadrifasciata, L. virens, M. minor, M. sartor, M. sutor, P. lurida, Rh. inquisitor, S. rubra, T. castaneum, C. quadripunctata, Ph. undulata, P. sericea, C. sylvicola, L. glabrirostris, O. coecus, O. multipunctatus, O. tenebricosus, A. polonicus, D. lineare, M. bipustulatus, Oe. virescens, G. stercorosus, O. thoracica, S. carinata, Ph. decorus.

The abundant species of the high mountain pastures are E. clathratus, L. quadrifasciata, L. virens, M. minor, M. sartor, M. sutor, O. cursor, Rh. inquisitor, S. rubra, T. castaneum, H. modeeri, L. caprea, 
O. cacaliae, O. intricata, O. virgulata, C. septempunctata, O. coecus, Ph. maculicornis, G. spiniger, O. ormayi, Ph. decorus.

Alpine meadows are inhabited by the next abundant species C. caraboides, C. sylvestris, N. transsylvanica, E. clathratus, O. cursor, O. cacaliae, O. intricata, O. virgulata, O. singularis.

While the richness and the diversity of Coleoptera species vary widely, functional diversity is stable in ecosystems and is more conservative. This indicates ecosystems capacity, complexity, and resilience (Sumarokov, 2009). Functional diversity describes ecosystem processes and the role of sole species or group of species in the ecosystem, such as the transmission of energy through trophic networks, diversification of ecological niches, and predicting of ecosystem dynamics (Petchey, Gaston, 2006). We analyze the trophic subdivision of Coleoptera in studied habitats (Fig. 4). First of all, we found that herbivorous Coleoptera predominates on carnivorous in the meadow habitats. They share $46 \%$ on steppes, $41 \%$ on high mountain pastures, and $42 \%$ on alpine meadows. These explained by the availability of a high variety of fodder herbaceous plants. In the forest habitats, however, herbivorous species constitute only near $20 \%$ of identified Coleoptera. Instead, carnivorous species predominate (41-47\%) in forest habitats, and, surprisingly, on alpine meadows (42\%). Contrary to meadows, in forest habitats it is a large proportion of xylophagous (11-17\%) and mycetophagous (13-15\%) Coleoptera. The proportion of detritophagous, necrophagous, and myxophagous species little vary within studied habitats. The total proportion of all types decompositors of dead organic (including mycetophagous species) consists of 16-19\% for meadow habitats and $34-37 \%$ for the forest habitats. It should be noted that the comparatively large amount (12\%) of commensal beetles in the nests of social insects is typical for the steppe habitats.

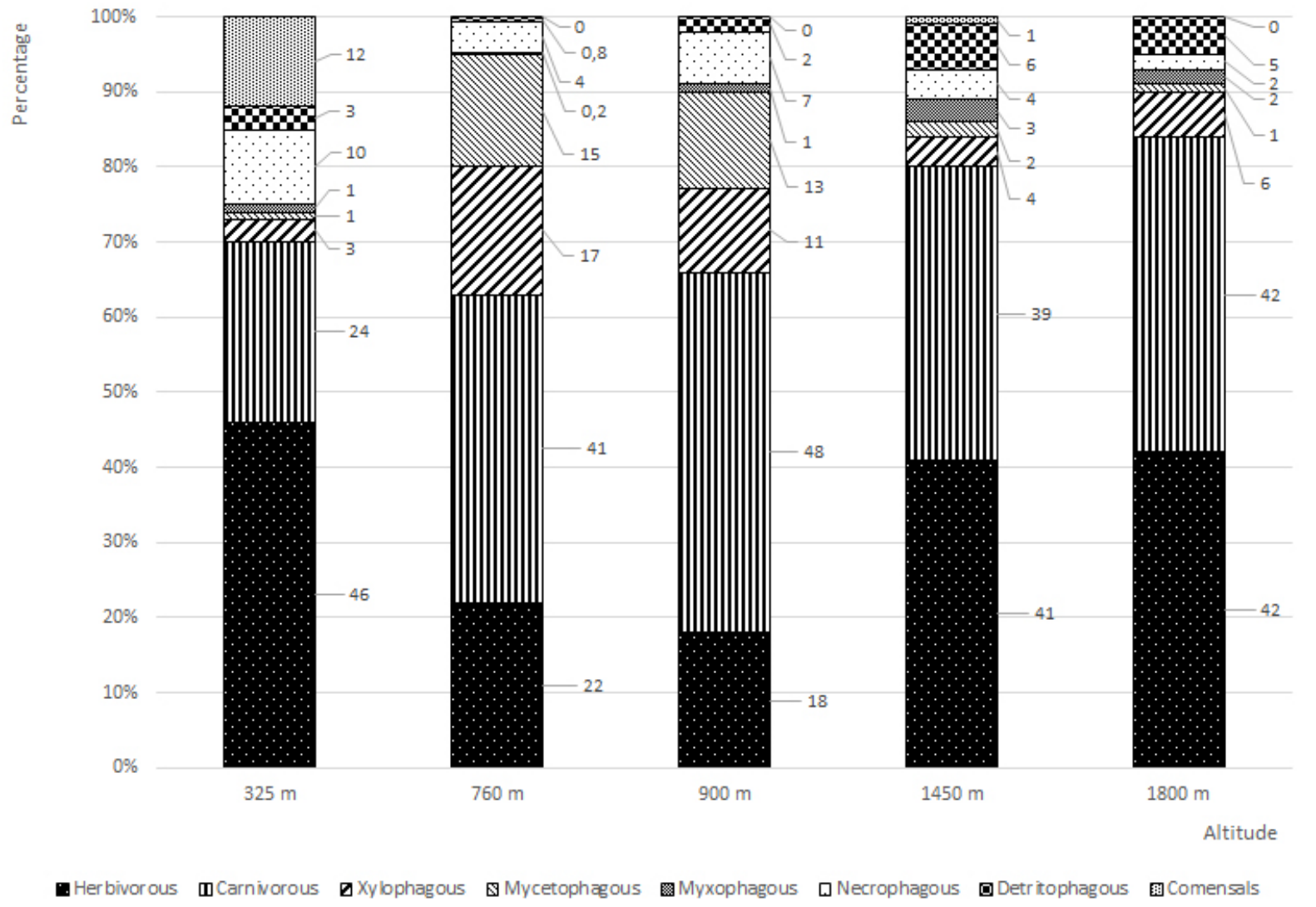

F i g . 4 Trophical composition of Coleoptera fauna.

Thus, two different types of Coleoptera-mediated transmission of energy have been identified in meadow and forest habitats. The first is typical of meadows and represents the producer-consumer type of energy transmission (living plants-phytophagous-lower carnivorous-higher carnivorous-saprotrophs). The second is typical of forests and represents the reducer-consumer type of energy transmission (dead plants debris and fungisaprotrophs-lower carnivorous-higher carnivorous-saprotrophs). We found that role of predation within Coleoptera in the primeval forest is slightly higher than in the secondary forest. This may indicate higher ecosystems capacity and deeper Coleoptera niche differentiation. 
Conservation consequences. Of the 595 species of identified Coleoptera, we found 344 species in Carpathian NP, 327 species in Halych NP, and 192 species in Gorgany NR. The most representative of studied Coleoptera fauna is in Carpathian NP, because there are all altitudinal vegetation belts. The beetle fauna of alpine meadows stands out sharply from the general forest matrix. The area of alpine meadows is very small and restricted only to Chornohora range in The Eastern Carpathians. Similarly, the steppe habitats are restricted by agricultural fields in the valley of the Dnister River and additionally, they are very fragmented (Zamoroka et al., 2018). Both types of habitats are rare and characterized by a large amount of Coleoptera species that do not occur in any other habitat within the region. Their fauna is vulnerable to the amount of anthropogenic and natural threats. These include land usage, recreation activity, the current climate changes, as well as spontaneous successions. While all alpine meadows are under protection, only a small part of the steppe habitats is protected (Zamoroka et al., 2018). Thus it needs to be a stricter limitation of usage of those habitats.

C o n c l u s i o n s. In summary, our results demonstrated that the Coleoptera fauna of the protected areas in Eastern Carpathians is highly diverse. We identified 595 species of beetles from 37 families within five types of habitats in two National Parks and one Nature Reserve. The general species richness gradually decreases from 327 species at an altitude of $325 \mathrm{~m}$ a. s. l. to 48 species at 1,800 $\mathrm{m}$ a. s. l. The sylvatic species of Coleoptera are predominating through the territory except alpine meadows in Chornohora range and steppes in the Dnister River valley. Coleoptera fauna is highly differentiated with a low similarity within habitats. We identified two different types of Coleoptera-mediated transmission of energy in meadow and forest habitats including the producerconsumer and the reducer-consumer energy transmission. The most vulnerable Coleoptera fauna is in habitats of alpine meadows and steppes due to land usage, recreation activity, climate changes, and spontaneous successions.

A c k n o w l e d ge me n t s. We wish to thank all people who helped us in identification of the collected materials. In particular, to Dr. O. F. Bartenev (Vasyl Karazin Kharkiv National University), Dr. I. A. Belousov (All-Russian Institute of Plant Protection), Dr. L. S. Cherniey (I. I. Schmalhausen Institute of Zoology of NAS of Ukraine), Dr. V. O. Chumak (Uzhhorod National University), Dr. V. G. Dolin (I. I. Schmalhausen Institute of Zoology of NAS of Ukraine), Mr. O. M. Drogvalenko (Museum of Nature of the Vasyl Karazin Kharkiv National University), Dr. B. M. Kataev (Zoological Institute of RAS), Dr. A. G. Kirejtchuk (Zoological Institute of RAS), Dr. V. V. Martynov (Donetsk National University), Dr. G. S. Medvedev (Zoological Institute of RAS), Dr. V. Yu. Nazarenko (I. I. Schmalhausen Institute of Zoology of NAS of Ukraine), Dr. A. A. Petrenko (I. I. Schmalhausen Institute of Zoology of NAS of Ukraine), Dr. V. B. Rizun (State Museun of Natural History of NAS of Ukraine); Dr. M. Ye. Sergeyev (Donetsk National University). We also wish to thanks people who supported our expeditions, accompanied and hosted us. In particular, we are very grateful to Dr. O. I. Kyseliuk (Carpathian National Park), Dr. V. B. Malaniuk (Halych National Park), Dr. V. I. Parpan (Vasyl Stephanyk Precarpathiam National University), Dr. O. M. Slobodian (Nature Reserve Gorgany), Mr. R. M. Bidychak (Vasyl Stephanyk Precarpathiam National University), Mr. V. V. Buchko (Halych National Park), Mr. V. M. Kysliak (Nature Reserve Gorgany), Mr. V. B. Tymochko (Carpathian National Park). In addition, we are grateful to Mr. O. M. Drogvalenko (Museum of Nature of the Vasyl Karazin Kharkiv National University) and Mr. D. V. Vovk (National Scientific Center 'Institute of Experimental and Clinical Veterinary Medicine') for their assistance in preparing the manuscript.

\section{R E F E R E N C E S}

Alonso-Zarazaga, M. A., Barrios, H., Borovec, R., Bouchard, P., Caldara, R., Colonnelli, E., Gültekin, L., Hlavač, P., Korotyaev, B., Lyal, C. H. C., Machado, A., Meregalli, M., Pierotti, H., Ren, L., Sanchez-Ruiz, M., Sforzi, A., Silfverberg, H., Skuhrovec, J., Tryzna, M., Velazquez de Castro, A. J., Yunakov, N. N. 2017. Cooperative Catalogue of Palaerctic Coleoptera Curculionoidea. Sociedad Entomológica Aragonesa, Zaragoza, 1-729. URL: http://sea-entomologia.org/monoelec.html. LSID: urn:lsid:zoobank.org: pub:911EF526-33F0-4970-8EC2-A7F5AC1E1D3D.

Audisio, P., Alonso Zarazaga, M., Slipinski, A., Nilsson, A., Jelínek, J., Taglianti, A., Turco, F., Otero, C., Canepari, C., Kral, D., Liberti, G., Sama, G., Nardi, G., Löbl, I., Horak, J., Kolibac, J., Háva, J., Sapiejewski, M., Jäch, M., Bologna, M., Biondi, M., Nikitsky, N., Mazzoldi, P., Zahradnik, P., Wegrzynowicz, P., Constantin, R., Gerstmeier, R., Zhantiev, R., Fattorini, S., Tomaszewska, W., Rücker, W., Vazquez-Albalate, X., Cassola, F., Angelini, F., Johnson, C., Schawaller, W., Regalin, R., Baviera, C., Rocchi, S., Cianferoni, F., Beenen, R., Schmitt, M., Sassi, D., Kippenberg, H., Zampetti, M., Trizzino, M., Chiari, S., Carpaneto, G., Sabatelli, S., de Jong, Y. 2015. Fauna Europaea: Coleoptera 2 (excl. series Elateriformia, Scarabaeiformia, Staphyliniformia and superfamily Curculionoidea). Biodiversity Data Journal, 3, e4750. DOI: https://doi.org/ 10.3897/BDJ.3.e4750.

Brovdiy, В. М. 1977. Fauna of Ukraine. Volume 19: Beetles. Issue 16: Leaf Beetles. Chrysoтеlinaе [Фауна Украӥни. Том 19: Жуки. Випуск 16: Жуки-листоїд. Хризомеліни]. Naukova Dumka, Kyiv, 1-384. [in Ukrainian].

Chumak, V., Duelli, P., Rizun, V., Obrist, M. K., Wirz, P. 2005. Arthropod biodiversity in virgin and managed forests in Central Europe. Forest Snow and Landscape Research, 79(1-2), 101-109. URL: https://www.dora.lib4ri.ch/wsl/islandora/object/wsl:15330. 
Dariusz, I., Löbl, I., eds. 2020. Catalogue of Palaearctic Coleoptera. Vol. 5: Tenebrionoidea. Revised and updated edition. Brill, Leiden, Boston, 1-945. DOI: https://doi.org/10.1163/9789004434998.

Dolin, V. H. 1966. To the knowledge of fauna and ecology of click beetles (Coleoptera, Elateridae) in Ukrainian Carpathians [До фауни та екології жуків-коваликів (Coleoptera, Elateridae) Українських Карпат]. In: Insects of Ukrainian Carpathians and Zakarpattia [Комахи Украӥнських Kарпат та Закарпаття]. Naukova Dumka, Kyiv, 38-44. [in Ukrainian].

Drinkwater, E., Robinson, E. J. H., Hart, A. G. 2019. Keeping invertebrate research ethical in a landscape of shifting public opinion. Methods in Ecology and Evolution, 10(8), 1-9. DOI: https://doi.org/10.1111/2041-210X.13208.

Fasulati, K. K. 1971. The Field Studies of Terrestrial Invertebrates [Полевое изучение наземных беспозвоночных]. Vysshaya Shkola, Moscow, 1-424. [in Russian].

Fischer, B., Larson, B. M. H. 2019. Collecting insects to conserve them: a call for ethical caution. Insect Conservation and Diversity, 12(3), 173-182. DOI: https://doi.org/10.1111/icad.12344.

Ghilarov, M. S. 1941. Methods for quantifying soil fauna [Методы количественного учёта почвенной фауны]. Реdology [Почвоведение], 4, 48-77. URL: https://www.elibrary.ru/item.asp?id=21627983. [in Russian].

Grootaert, P., Pollet, M., Dekoninck, W., van Achterberg, C. 2010. Chapter 15 - Sampling insects: general techniques, strategies and remarks. In: Eymann, J., Degreef, J., Häuser, Ch., Monje, J. C., Samyn, Y., VandenSpiegel, D., eds. Manual on Field Recording Techniques and Protocols for All Taxa Biodiversity Inventories and Monitoring. Abc Taxa, 8(2), 377-399. URL: http://www.abctaxa.be/volumes/volume-8-manual-atbi/volumes/volume-8-manual-atbi/chapter-15/Chapter_15.pdf.

Hryhora, I. M., Solomakha, V. A. 2005. Vegetation of Ukraine (Ecological-Coenotic, Floral and Geographical Essay) [Pocлиннicmь Украйни (еколого-цеенотичний, флористичний та географічний нарис)]. Fitosotsiotsentr, Kyiv. ISBN: 9663060107, 1-452. [in Ukrainian].

Kajtoch, L., Lachowska-Cierlik, D., Mazur, M. 2009. Genetic diversity of the xerothermic weevils Polydrusus inustus and Centricnemus leucogrammus (Coleoptera: Curculionidae) in central Europe. European Journal of Entomology, 106(3), 325-334. DOI: https://doi.org/10.14411/eje.2009.040.

Krivosheyev, R. Ye. 2012. Annotated list of the short-winged mold beetles (Coleoptera: Pselaphidae) of the State Museum of Natural History, NAS of Ukraine (Lviv) [Анотований список жуків-потаємців (Сoleoptera: Pselaphidae) Державного природознавчого музею НАН України (Львів)]. Ukrainska Entomofaunistyka [Украӥнська ентомофауністика], 3(3), 5-13. URL: https://sites.google.com/site/ukraienskaentomofaunistikaua/contents-1/pdfs_2/Ukrentfau 3_3_2_Krivosheyev.pdf. [in Ukrainian].

Kubisz, D., Kajtoch, L., Mazur, M., Rizun, V. 2012. Molecular barcoding for central-eastern European Crioceris leaf-beetles (Coleoptera: Chrysomelidae). Central European Journal of Biology, 7(1), 69-76. DOI: https://doi.org/10.2478/s11535-011-0099-4.

Kuntze, R., Noskiewicz, J. 1938. Zarys zoogeografii polskiego Podola. Prace Naukowe Wydawnictwo Towarzystwa Naukowego we Lwowie, 2(4), 1-538.

Lazorko, V. 1963. Materials to Systematics and Faunistics of Beetles of Ukraine [Матеріяли до систематики і фавністики жуків України]. The Shevchenko Scientific Society, Vancouver, Canada, 1-123. [in Ukrainian].

Löbl, I., Löbl, D., eds. 2015. Catalogue of Palaearctic Coleoptera. Vol. 2: Hydrophiloidea - Staphylinoidea. Revised and updated edition. Brill, Leiden, Boston, 1-1702. DOI: https://doi.org/10.1163/9789004296855.

Löbl, I., Löbl, D., eds. 2016. Catalogue of Palaearctic Coleoptera. Vol. 3: Scarabaeoidea - Scirtoidea - Dascilloidea - Buprestoidea Byrrhoidea. Revised and updated edition. Brill, Leiden, Boston, 1-983. DOI: https://doi.org/10.1163/9789004309142.

Löbl, I., Löbl, D., eds. 2017. Catalogue of Palaearctic Coleoptera. Vol. 1: Archostemata - Myxophaga - Adephaga. Revised and updated edition. Brill, Leiden, Boston, 1-1443. DOI: https://doi.org/10.1163/9789004330290.

Löbl, I., Smetana, A., eds. 2007. Catalogue of Palaearctic Coleoptera. Vol. 4: Elateroidea - Derodontoidea - Bostrichoidea Lymexyloidea - Cleroidea - Cucujoidea. Apollo Book, Stenstrup, 1-935. DOI: https://doi.org/10.1163/9789004260894.

Löbl, I., Smetana, A., eds. 2010. Catalogue of Palaearctic Coleoptera. Vol. 6: Chrysomeloidea. Apollo Book, Stenstrup, 1-924. DOI: https://doi.org/10.1163/9789004260917.

Lomnicki, M. A. 1884. Catalogus Coleopterorum Haliciae. Sumptibus L. Zontaki, Custodis Musaei Dzieduszyckiani, Leopoli, 1-43. URL: https://rcin.org.pl/dlibra/doccontent?id=57366.

Lomnicki, M. A. 1913. Wykaz chrząszczów czyli Tęgopokrywych (Coleoptera) ziem polskich. (Catalogus coleopterorum Poloniae). Kosmos. Seria A - Biologia, 38, 21-155. URL: https://baza.biomap.pl/en/getpdf/5282_Lomnicki_MA_1913.pdf.

Malynovskyi, K. A., Krichfalushiy, V. V. 2000. Vegetation of Ukraine. High Mountain Vegetation. Volumе 1. [Рослинність України. Високогірна рослинність. Том 1]. Fitosotsiotsentr, Kyiv, 1-231. ISBN: 9667459780. [in Ukrainian].

Mateleshko, O. Yu. 2008. Water Beetles of the Ukrainian Carpathians [Водні твердокрилі Українських Kарпат]. Mystetska liniia, Uzhhorod, 1-200. ISBN: 9789668764684. DOI: https://doi.org/10.13140/RG.2.1.3470.9365/1. [in Ukrainian].

Nikulina, T., Mandelshtam, M., Petrov, A., Nazarenko, V., Yunakov, N. 2015. A survey of the weevils of Ukraine. Bark and ambrosia beetles (Coleoptera: Curculionidae: Platypodinae and Scolytinae). Zootaxa, 3912(1), 1-61. DOI: https://doi.org/10.11646/ zootaxa.3912.1.1.

Nowicki, M. 1873. Verzeichniss galizischer Käfer. In: Beitrage zur Insektenfauna Galiziens. Jagiellonian University, Krakow, 7-52.

Ocheretna, K. 2020. Silken-fungus beetles (Cryptophagidae, Coleoptera) of the Ukrainian Carpathians. Geo\&Bio, 19, 104-120. DOI: https://doi.org/10.15407/gb1911.

Petchey, O. L., Gaston, K. J. 2006. Functional diversity: back to basics and looking forward. Ecology Letters, 9(6), 741-758. DOI: https://doi.org/10.1111/j.1461-0248.2006.00924.x.

Petrenko, A. A. 2009. Staphylinidae. In: Prykhodko, M. M., Kyseliuk, O. I., Yavorskyi, A. I., eds. Carpathian National Nature Park [Карпатський національний природний парк]. Foliant, Ivano-Frankivsk, 539-552. ISBN: 9789662988192. [in Ukrainian].

Petrenko, A. A., Nadvornyy, V. G. 1990. The rove beetles as indicators of biocenoses' state in Carрathian Reserve [Жуки-стафилиниды индикаторы состояния биоценозов Карпатского заповедника]. Reserves of the USSR - Their Present and Future: Abstracts of the All-Union Conference [Заповедники СССР-их настоящеее и будущее: тезисы докладов всесоюзной конференции], 3 , 112-114. [in Russian].

Rizun, V. B. 2003. The Ground Beetles of the Ukrainian Carpathians [Tуруни Українських Kapnam]. Lviv, 1-207. ISBN: 9660215398. [in Ukrainian].

Rizun, V. B., Tymochko, V. B., Chumak, V. O. 2004. Communities of the ground beetles (Coleoptera, Carabidae) in beech and fir forests of the Carpathian National Nature Park [Угруповання жуків-турунів (Coleoptera, Carabidae) букових та ялицевих лісів Карпатського національного природного парку]. Scientific Bulletin of the Uzhhorod University. Series: Biology [Hayковий вісник Ужгородського університету. Серія: Біологія], 14, 34-43. [in Ukrainian]. 
Steiner, A., Häuser, Ch. 2010. Chapter 16 - Recording insects by light-traps. In: Eymann, J., Degreef, J., Häuser, Ch., Monje, J. C., Samyn, Y., VandenSpiegel, D., eds. Manual on Field Recording Techniques and Protocols for All Taxa Biodiversity Inventories and Monitoring. Abc Taxa, 8(2), 400-422. URL: http://www.abctaxa.be/volumes/volume-8-manual-atbi/volumes/volume-8-manualatbi/chapter-16/Chapter_16.pdf.

Stork, N. E., McBroom, J., Gely, C., Hamilton, A. J. 2015. New approaches narrow global species estimates for beetles, insects, and terrestrial arthropods. Proceedings of the National Academy of Sciences of the United States of America, 112(24), 7519-7523. DOI: https://doi.org/10.1073/pnas.1502408112.

Sumarokov, A. M. 2009. Renewals of Biotic Potential of Biogeocenoses at Diminishing of the Pesticidal Loadings [Восстановление биотического потенциала биогеоченозов при уменьшении пестицидных нагрузок]. Veber, Donetsk, 1-193. ISBN: 9789663352695. [in Russian].

Tveritina, T. A. 1959. Weevils of the Transcarpathian Region [Долгоносики Закарпатской области]. Scientific Notes of Uzhgorod University [Научные записки Ужгородского университета], 40, 181-187. [in Russian].

Yanytsky, T. P. 2001. Jewel Beetles (Coleoptera, Buprestidae) of Western Ukraine [Жуки-златки (Coleoptera, Buprestidae) Західної України. PhD Thesis. The State Museum of Natural History of the National Academy of Sciences of Ukraine, Lviv, 1-163. [in Ukrainian].

Yanytsky, T. P. 2013. A checklist of the Western Ukrainian Buprestidae (Coleoptera). Scientific Notes of the State Museum of Natural History, 29, 173-180. URL: http://nbuv.gov.ua/UJRN/Nzdpm_2013_29_29.

Yunakov, N., Nazarenko, V., Filimonov, R., Volovnik, S. 2018. A survey of the weevils of Ukraine (Coleoptera: Curculionoidea). Zootaxa, 4404(1), 1-494. DOI: https://doi.org/10.11646/zootaxa.4404.1.1.

Zahaykevych, I. K. 1957. Materials to study of bark beetles' fauna in western regions of Ukraine [Матеріяли до вивчення фавни короїдів західних областей України]. Proceeding of the Zoological Museum of the Academy of Sciencies of Ukrainian SSR [3бірник праиь Зоологічного музею АН УССР], 28, 100-101. [in Ukrainian].

Zahaykevych, I. K. 1959. Contribution to study of the longhorn beetles (Cerambycidae) of Stanislav Region [До вивчення вусачів (Cerambycidae) Станіславської обл.]. In: Problems of Entomology in Ukraine [Проблеми ентомології на Україні]. Publishing House of the Academy of Sciencies of Ukrainian SSR, Kyiv, 45-47. [in Ukrainian].

Zahaykevych, I. K. 1978. Jewel beetles (Coleoptera, Buprestidae) [Твердокрилі родини златок (Сoleoptera, Buprestidae)]. In: Catalogues of Museum Collections: Collection of Scientific Works of the State Museum of Natural History of the Academy of Sciencies of Ukrainian SSR [Каталог музейних фондів: збірник наукових прачь Державного природознавчого музею АН УРСР]. Naukova Dumka, Kyiv, 210-229. [in Ukrainian].

Zamoroka, A. M. 2008. The longhorn beetles in the forest ecosystems of Carpathians and Precarpathia [Жуки-вусачі у лісових екосистемах Карпат і Передкарпаття]. Herald of Vasyl Stefanyk Precarpathian National University. Series Biology [Bicник Прикарпатського національного університету імені Василя Стефаника. Серія: Біологія], 10, 21-31. [in Ukrainian].

Zamoroka, A. M. 2009. Ecological Features of Entomocomplexes of the Longhorn Beetles (Coleoptera: Cerambycidae) in the Forest Ecosystems of Northern-East Macroslope of Ukrainian Carpathians [Екологічні особливості ентомокомплексів жуків-вусачів (Coleoptera: Cerambycidae) у лісових екосистемах північно-східного макросхилу Украӥнських Карпат]. PhD Thesis. Vasyl Stefanyk Precarpathian National University, Ivano-Frankivsk, 1-212. [in Ukrainian].

Zamoroka, A. M. 2018. The longhorn beetles (Coleoptera: Cerambycidae) of the Eastern Carpathian Mountains in Ukraine. Munis Entomology \& Zoology, 13(2), 655-691. URL: https://www.munisentzool.org/Issue/abstract/the-longhorn-beetles-coleopteracerambycidae-of-the-eastern-carpathian-mountains-in-ukraine-1224.

Zamoroka, A. M. 2019. A new subspecies of Dorcadion fulvum (Scopoli, 1763) (Coleoptera: Cerambycidae) from western Ukraine. Polish Journal of Entomology, 88(4), 363-378. DOI: https://doi.org/10.2478/pjen-2019-0024.

Zamoroka, A. M., Panin, R. Yu., Kapelukh, Y. I.. Podobivskiy, S. S. 2012. The catalogue of the longhorn beetles (Coleoptera: Cerambycidae) of western Podillya, Ukraine. Munis Entomology \& Zoology, 7(2), 1145-1177. URL: https://www.munisentzool.org/ Issue/abstract/the-catalogue-of-the-longhorn-beetles-coleoptera-cerambycidae-of-western-podillya-ukraine-614.

Zamoroka, A. M., ed., Shumska, N. V., Buchko, V. V., Dmytrash-Vatseba, I. I., Malaniuk, V. B., Smirnov, N.A. 2018. The Steppe Biote of Burshtyn Opillya [Біота лучних степів Бурштинського Опілля]. Symfoniia Forte, Ivano-Frankivsk, 1-212. ISBN: 9789662861372. [in Ukrainian].

Zamoroka, A. M., Semaniuk, D. V., Shparyk, V. Yu., Mykytyn, T. V., Skrypnyk, S. V. 2019. Taxonomic position of Anastrangalia reyi and A. sequensi (Coleoptera, Cerambycidae) based on molecular and morphological data. Vestnik Zoologii, 53(3), 209-226. DOI: https://doi.org/10.2478/vzoo-2019-0021.

Zawadzki, A. 1825. Rzut oka na osobliwości we względzie historyi naturalnej, widziane w podróży przedsięwziętej przez Karpaty Stryjskiego i Stanisławowskiego obwodu. Rozmaitości. Pismo Dodatkowe do Gazety Lwowskiej, 5(21), 161-163. URL: https://www.wbc.poznan.pl/dlibra/publication/210080/edition/185843.

Agro-association 'Chysta Krynytsia',

Vasyl Stefanyk Precarpathian National University 